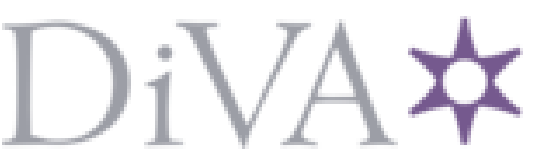

http://www.diva-portal.org

[This is not an article, chapter, of conference paper!] 


\title{
Contact fatigue initiation and tensile surface stresses at a point asperity which passes an elastohydrodynamic contact
}

\author{
Carl-Magnus Everitt ${ }^{*}$ and Bo Alfredsson ${ }^{1}$ \\ ${ }^{1}$ Department of Solid Mechanics, Royal Institute of Technology - KTH, 10044 Stockholm, \\ Sweden \\ * Corresponding author, cmev@kth.se
}

\begin{abstract}
Contact mechanics and tribology was combined with fundamental fatigue and fracture mechanics to form a new mechanism for surface initiated rolling contact fatigue. Following, fatigue was investigated numerically for single asperities and craters in lubricated rolling contact surfaces. The hypothesis suggests that asperity point contacts can create sufficiently large tensile stresses for fatigue. The investigated case corresponded to a heavily loaded truck gear with ground surfaces. Reynolds equation resolved the elastohydrodynamic effect of the asperity in the transient three dimensional contacts. The Findley critical plane criterion was used for multiaxial and non-proportional fatigue evaluation. The simulations confirmed the new mechanism for rolling contact fatigue and showed how asperities can create contact fatigue in the lubricated contacts even without slip.
\end{abstract}

\section{Keywords}

Spalling; Elastohydrodynamic; Fatigue; Contact mechanics. 


\section{Nomenclature}

Upper case letters are used for the dimensionless counterpart of lower case letters, except for the elastic module $E$ in Pa.

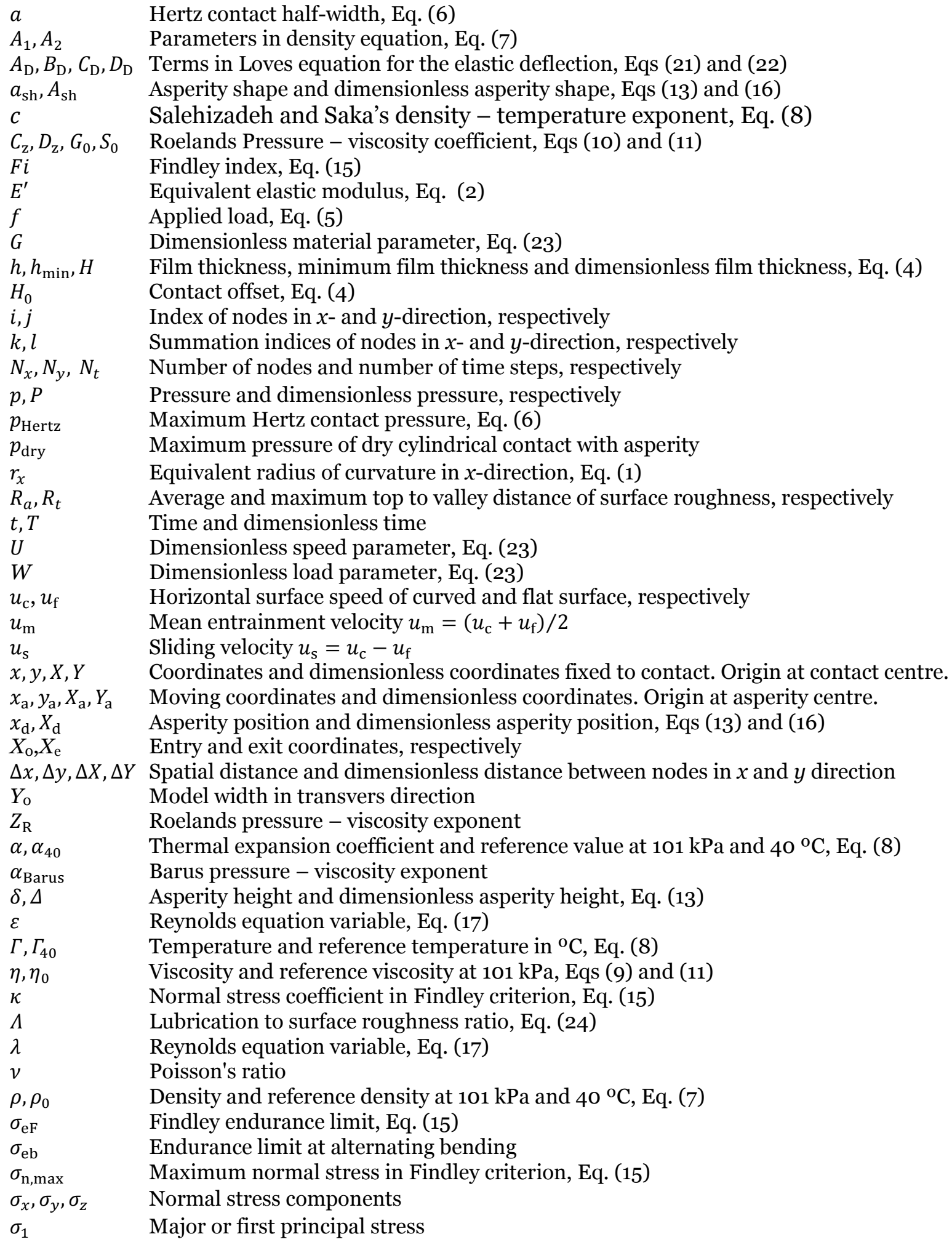




$\begin{array}{ll}\tau_{\mathrm{amp}} & \text { Shear stress amplitude in Findley criterion, Eq. (15) } \\ \tau_{x z}, \tau_{y z}, \tau_{x y} & \text { Shear stress components } \\ \omega_{x}, \omega_{y}, \Omega_{x}, \Omega_{y} & \text { Asperity half width in } x \text { and } y \text { direction, Eqs (13) and (16) } \\ & \text { Indicates the maximum value of the symbol }\end{array}$

\section{Introduction}

Gear and bearings surfaces may eventually fail due to rolling contact fatigue (RCF). A common failure mode of RCF is surface induced pitting, also called spalling, where small pieces of surface material are chipped off. In 1935 Way [1] did a thorough investigation on pitting and found that lubrication was a fundamental prerequisite for the damage. The Failure Atlas by Tallian [2] shows a multitude of typical RCF damages and describes the damage process from a phenomenological viewpoint. Although the damage development is reasonably well established, the explaining mechanism for the damage process is still discussed.

Pitting damages can be initiated at the surface or just below. This work focused on a mechanism for crack initiation of surface initiated spalling or pitting. Different definitions of the size and shape of surface initiated pitting has been presented by for example Way [1] and Tallain [2]. Fig. 1 shows two typical RCF pits in truck spur gears. The geometry and manufacturing process of the gears are described by MacAldener and Olsson [3]. The gear with the pit in Fig. 1a was loaded with a torque of $1850 \mathrm{Nm}$ and the gear with the pit of Fig. 1b was loaded with a torque of $1680 \mathrm{Nm}$. With help of the program Helical 3D from Ansol [4] the peak pressure, the entrainment velocity and the slip speed were determined. These are presented together with the original surface roughness, i.e. after manufacturing but before use, in Fig. 2a for positions along the gear tooth flank.

For the current analysis the important aspect was the recognition that the surface initiated $\mathrm{RCF}$ crater forms by a crack which initiate at one point in the surface. For both pits in Fig. 1, the cracks initiated at the lower tip of the pit. The crack then grew into the material and finally caused a piece of material to chip of, forming the pit. Since the cracks initiated at one point the initiation mechanism for the crack must be of local sort. The pitch line is visible in the photos as a bright horizontal reflection in the surface slightly below the middle of the pit. Most RCF pits in the test series were located close to the pitch line as in Fig. 1a, or at the pitch line such as in Fig. $1 \mathrm{~b}$.

Olsson [5] proposed a mechanism for RCF initiation based on the requirement of cyclic tensile stresses for crack initiation and growth. Note that cyclic compressive stresses with local cyclic plasticity may degrade the material but can not open a crack. Hence, for fatigue to develop the maximum principal stress on a crack plane has to be positive. Tensile surface stresses develop radially outside point type contacts, for instance at over-rolling of a point type asperity. The vital difference in surface stresses between normal point and normal line contacts was recognised. Not only does the point contact include stress variation in the third, transverse direction, the stress solution in the rolling plane is fundamentally different from that of the line contact, see Appendix A. The normal line contact only gives compressive stresses. The normal point contact on the other hand has a tensile radial surface stress which in the rolling plane will act in the rolling direction. Hence, normal line contacts will not open a crack and can therefore not explain fatigue. Stresses from normal point contacts may however initiate fatigue and will open surface cracks. Dahlberg and Alfredsson [6] investigated the surface stresses arising around an axisymmetric surface asperity over rolled 
by a cylinder. For dry conditions they concluded that the tensile stresses in front of the asperity are sufficiently large to initiate fatigue. Hannes and Alfredsson performed a series of fracture mechanical investigations [7], [8], [9] and [10] on the surface crack growth behaviour by the combined asperity and rolling cylinder contact load. They found that the crack behaviour can be predicted with respect to crack growth direction in the rolling cross-section [7], i.e. the pitting entry angle $\beta$ [7], the pitting life [8] and [9], and the surface view opening angle $\alpha_{\text {pit }}[10]$, of the archetypical sea-shell shaped pit crater exemplified in Fig. 1a.

The analyses in the literature by Dahlberg and Alfredsson [6] and [11] showed that for dry conditions sufficiently large in-surface stresses can develop at point type asperities to initiate RCF cracks both with and without slip. The pit in Fig. 1b developed at the pitch line where slip was small or absent. It illustrates that if a mechanism for surface initiated RCF should be generally applicable, then it must work also in the absence of contact traction. Negative slip, as is found in the dedendum of the pinion gear or along the centre lines in the outer ring of deep groove bearings, will enhance tensile stresses in front of the contact entering asperity but may not alone be the general root explanation for all RCF cracks. Instead the in-surface tensile stress from the point loaded asperity appears to be the important factor for RCF.

Fractographic investigation of surface distress [12] shows different crack entry angles to the surface at the pitch line compared to the dedendum with negative slip and the addendum with positive slip on the pinion gear teeth. At the pitch line with pure rolling the crack entry angle $\beta$ $=18-28{ }^{\circ}$ whereas in the dedendum $\beta=41-50^{\circ}$. Purified point contact experiments [13] showed the importance of point contact load and friction on the surface distress crack entry angle. The pits in Fig. 1 were in fact located at, or near, the pitch line where slip was absent or small. The entry angle $\beta=23-30^{\circ}$ for these and similar pits [7] verifies that slip in this case was small or absent which motivates detailed investigation of the contact and stress state at pure rolling when focus of the investigation is the understanding of the underlying mechanism for RCF.

At the pitch line slip is absent and only the point load can give tensile surface stresses. The size of these will be decisive for verifying the asperity point load mechanism. Further down in the addendum slip may add tensile stresses from friction, which would complicate the possibilities to draw important conclusions. Therefore, the condition at the pitch line was here studied in detail. 

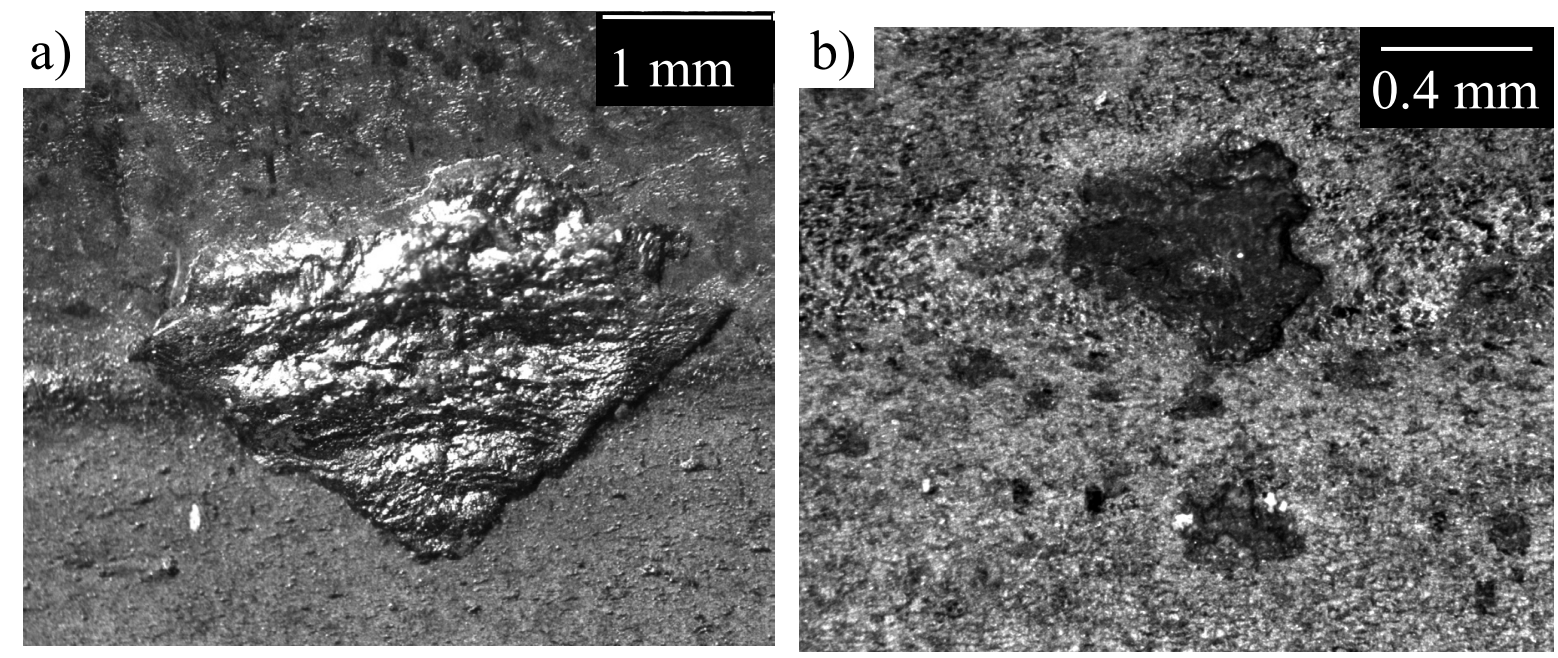

Fig. 1. Surface views of two pits in spur gear teeth. a) An archetypical sea-shell pit. b) A pit which initiated at the pitch line.

Compared to the earlier investigations [6] to [11] on the asperity point load mechanism, the present work focused on the effects the lubrication has on the surface stresses at over-rolled asperities or craters, including the risk for fatigue initiation. Since the investigation focused on the underlying mechanism which creates surface initiated RCF and therefore must be able to predict RCF at pure rolling, it was limited to the conditions close to the pitch line or rolling point where pure normal entanglement prevails. Pure, normally directed, line loads of any shapes do not yield tensile stresses in the surface. However, when a point asperity or point crater passes through the contact, it will give rise to a point contact load which yields radial surface tension, see the stress solutions for line and point loads in Appendix A. The amplitude of the point load from the irregularity depends on the magnitude of the line load, the asperity geometry and relative surface compliances at the asperity contact.

The current gear cylinder contact, with an asperity, was modelled as the equivalent elastic cylinder with the axisymmetric asperity in Fig. $2 \mathrm{~b}$ rolled against a flat rigid surface. The asperity is compared with the real surface roughness profile in the bottom curve of Fig. 2a. The profile was measured in the rolling direction. Note that the roughness is fairly constant throughout the full length of the contact. The origin was located in the symmetry plane of the asperity and at the centre of the cylinder contact. The $x$-axis was oriented in the rolling direction. The $y$-axis was oriented in the transverse rolling direction and the $z$-axis was directed into the material of the curved surface. The asperity position was defined by the parameter $x_{\mathrm{d}}$ which changed for each time step. At the centre of the asperity, a material fixed coordinate system was defined, $x_{\mathrm{a}}=x-x_{\mathrm{d}}, y_{a}=y$ and $z_{a}=z$. This coordinate system moved with the material. In Fig. $2 \mathrm{~b}$ the asperity is on the exit side of the contact since $x_{\mathrm{d}}$ is defined in the same direction as $x$, which is opposite the rolling direction (RD). Hence, the rolling direction and the contact movement are towards the left in Fig. $2 b$ in a room fix view and the asperity move to the right in the figure in a contact following view. 

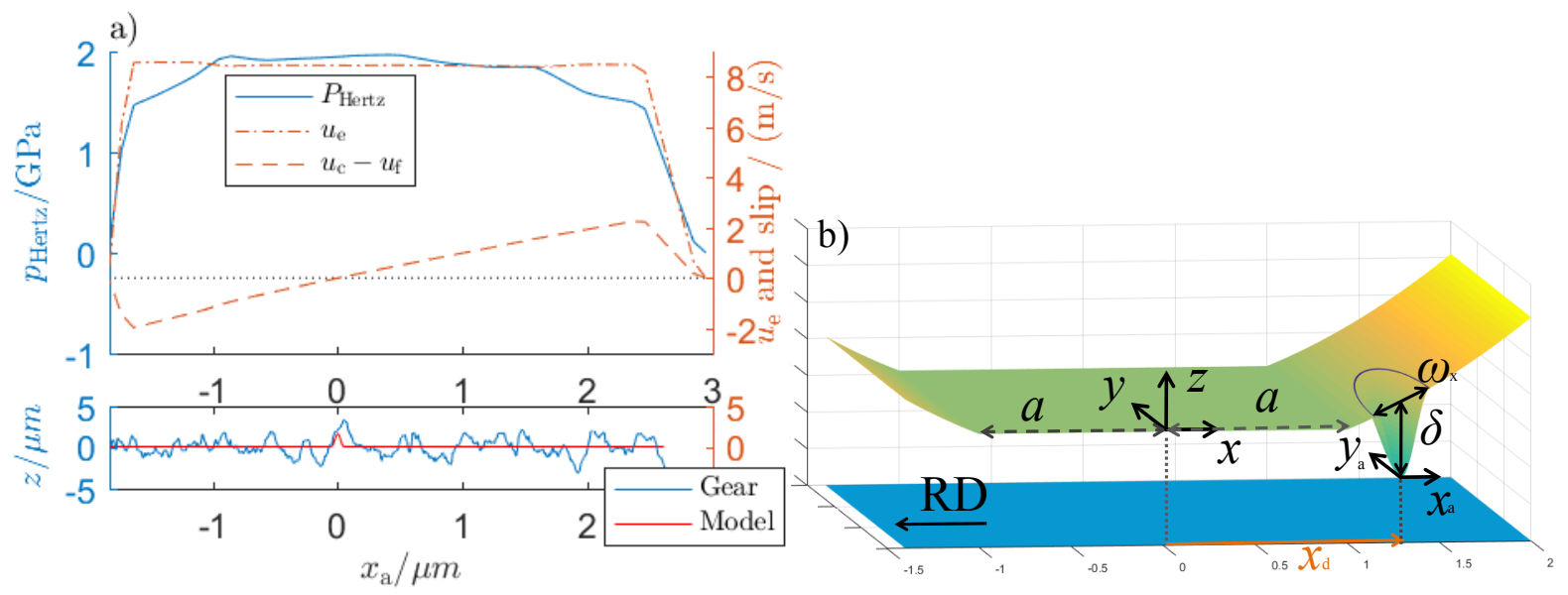

Fig. 2. a) Load conditions and surface roughness of the gear tooth. b) The present equivalent contact geometry with the asperity positioned at the contact exit, see text for details.

At highly loaded rolling Hertzian contacts with lubrication, so called elastohydrodynamic lubrication (EHL) conditions prevails. Already at pure rolling EHL conditions, the normal contact pressure distributions differ from the Hertzian pressure distribution. The most pronounced differences are the pressure spike at the contact exit and small pressure increase just before the Hertzian contact starts at the contact entry. The lubrication effects on the overall pressure, will affect the asperity load and thereby also the surface stresses near the contacting asperity.

\section{$1.1 \quad$ Numerical calculations}

EHL contacts are well described by the Reynolds equation which gives the pressure distribution and load carrying capacity of thin lubricating films. In 1945, Ertel combined the elastic deformation of the contact surfaces with the pressure dependent properties of the lubricant [14] [15].

An early numerical model for EHL based on sub-division of the pressure zone was developed by Petrusevich in 1951 [16] and Dowson and Higginson in 1959 [17] for cylindrical contacts. Dowson and Higginson used their model to derive an equation to estimate the minimum film thickness of a contact based on the three non-dimensional parameters $W, G, U$ [18]. In 1976 Hamrock and Dowson [19] solved the EHL for a circular contact. Current studies aim at including more aspects of the contact to get a better understanding of the contact and to yield more accurate results. An in depth review of the EHL history was presented by Dowson and Ehret's in 1999 [20]. There exist later reviews on EHL which describe later research developments, for example Spikes 2006 [21] and Lugt and Morales 2011 [22].

EHL simulations are highly non-linear due to the interactions between the pressure, the surface deformations and the flow of the lubrication. The elastic deformation affects the shape of the lubrication flow which in turn affects the load carrying capacities of the setup. The high contact pressure yields a large non-linear increase of the viscosity. To manage these non-linarites the EHL contact is solved with an iteration scheme where the Reynolds equation is solved simultaneously with the elastic deformation, a pressure viscosity equation, a pressure density equation and a force balancing equation. To improve convergence while keeping a stable procedure, a combination of underrelaxed Jacobi and underrelaxed Gauss- 
Seidel methods are often used to solve EHL problems. An informative description of the numerical implementation is presented by Venner [23].

One research area that has drawn increasing interest is the importance of surface roughness and asperities on the EHL contacts. For instance, Masjedi and Khonsari extended the formulas of central and minimum film thickness to include load sharing between the lubricant and the asperities [24]. Rosenkranz et al. demonstrate that pits can reduce the coefficient of friction [25]. Šperka et al. [26] showed experimentally that sliding will separate two different effects that surface irregularities have on the contact. One is the irregularity itself and the other is the so called complementary effect (CE) which is the effect the irregularity has on the lubrication film in the inlet. The CE will travel with the speed of the lubrication instead of the speed of the irregularity. Křupka et al. showed with experiments that point and line indents can cause lubrication breakdown due to CE effect [27]. Křupka et al. states that the ratio between the minimum lubrication height and the surface roughness, called the lambda ratio, is not alone sufficient to always determine the lubrication regime and the fatigue risk. However, when the surfaces break through the lubrication, or are close to, the risk of surface damage increases.

\subsection{Damage simulations in EHL}

To decrease frictional losses of gear components the viscosity should be as low as possible. When the viscosity is decreased so is the film thickness which yields greater influences of the surface roughness. The influence of the surface roughness has been studied for some time. An early review on the subject was done by Elrod in 1977 [28]. Since then, work has been done by among others Cheng and Bali [29] who published numerical results showing that ridges and furrows have a significant impact on the EHL pressure distribution and therefore also on the stresses in the material for line contacts with line defects. Lubrecht et al. [30] studied the life reduction from asperities and dents. They considered the hydrostatic stress component for dry line contacts with transverse ridges and grooves or spherical contacts with asperities and dents. Höln et al. [31] presented a detailed experimental investigation on the effects of the surface roughness on the lubrication shape and the pressure distributions for line contacts with line roughness, i.e. ridges and furrows, or grooves.

In recent years many models have been developed for simulating mixed EHL contacts, for example by Epstein et al. [32], Ren et al. [33] and Li and Kahraman [34]. Ren et al. investigated how the surface roughness affects the fluid flow in the contact and found that transverse roughness yield larger fluid film than longitudinal roughness. Epstein et al. evaluated roughness induced fatigue based on an effective stress with both the model developed by Ioannides and Harris [35] and the model by Zaretsky [36]. Zhu et al. [37] used von Mises stress to estimate the pitting life for contacts with 2 dimensional rough surfaces. Qiao et al. [38] compared some fatigue criteria for a line type rough contact. Xu et al. [39] considered the von Mises stress for dents in spherical contacts and found that the maximum effective stress approached the surface. Jiang et al. [40] and Deolaliker [41] et al. studied the contact pressure and temperature increase due to asperities passing through the EHL contacts. Hooke et al. [42] presents a new numerical set-up for EHL simulation including asperities. Li and Kahraman did also look into fatigue caused by the roughness but instead with the fatigue model proposed by Liu and Mahadevan [43]. All point out roughness as an important parameter for pitting and state good agreement with experiments where higher pressures and rougher surfaces display shorter life and higher risk for pitting. Nélias and Ville [44] presents experiments where local dents produced RCF. Their analysis is however 2 dimensional with line loads on grooves and only considering the risk for plastic deformation 
by presenting $\tau_{\max }$, i.e. half the maximum Tresca effective stress, during over rolling. Morales-Espejel and Gabelli [45] performed RCF experiments with dents and simulated the pitting evolution by removing material at points with high fatigue prediction by the Dang Van criterion.

In a review from 2014 Morales-Espejel [46] describes the progress of the understanding of the influences of surface roughness on the behaviour of micro pitting. The review also shows how small hard inclusions can, when loaded, generate bumps, which behave similar to ridges in EHL contacts. Other studies, such as experiments by Ahlroos et al. [47] and the 2 dimensional numerical simulations of cylindrical gear contacts with ridges and slip by Sharif et al. [48] and Qiao et al. [38] show that the damage increases with decreased lambda-ratio. These studies highlight surface roughness as important for rolling contact fatigue initiation. However, since they only consider line contacts with line roughness and line ridges they cannot explain why the pits in Fig. 1 are limited in the transverse direction, why and how they develop at specific positions and extends in the v-form to the sea-shell shape.

The purpose of the present work was to identify and quantify how the tensile in-surface stresses at and around the asperity or crater initiate RCF in the surface when they pass a contact with pure rolling EHL conditions. Contact mechanics theory and the numerical treatment of EHL were combined with fundamental understanding of fatigue crack initiation and fatigue crack propagation in hardened steel to form the asperity point load mechanism which here should be verified for EHD conditions. Furthermore, the goal was to determine surface and lubrication conditions for which these stresses can create surface initiated RCF.

Instead of investigating general rough surfaces, the work focused on the in-surface stress field around one single smooth axi-symmetric irregularity. The focus on individual well defined asperities and dents contributed to verifying the asperity point load mechanism for RCF and provided important understanding on the underlying mechanism and damage process for $\mathrm{RCF}$. The fatigue risk at and around the irregularity was evaluated according to the Findley criterion [49], since it is a widely tested and used fatigue criterion which has shown to work well for contact situations [50], [51]. The evaluation was performed for asperities undergoing contact conditions in a highly loaded truck spur gear. The results show, in accordance with the literature, that asperities may cause fatigue damage but the present results also show how the asperity causes the stresses which may initiate the pit creating crack. The work includes results for a range of temperatures, irregularity shapes and oil parameters for which irregularities may give rise to sufficiently large stresses to cause fatigue. The interaction between smooth craters, such as dents, and the EHL was investigated with respect to the surface stresses inside and surrounding the dent.

\section{Theoretical background}

The rolling gear teeth, or roller bearing, contact was converted to an equivalent contact with a cylinder rolling on a flat surface, see Fig. $2 b$. The shapes of the real application contacts are often somewhat elliptical, but since the transverse length of the contact is much greater than the contact width, the cylinder approximation was assumed applicable for the central part of the gear contact. The flat surface was modelled as rigid with the combined elasticity of both surfaces included in the cylinder material. The cylinder was given the equivalent radius

$$
r_{\mathrm{x}}=\frac{r_{1} r_{2}}{r_{1}+r_{2}}
$$


where $\mathrm{r} 1$ and $\mathrm{r} 2$ are the radii of curvature of the respective surfaces. The equivalent elastic modulus was

$$
E^{\prime}=\frac{2 E_{1} E_{2}}{\left(1-v_{2}^{2}\right) E_{1}+\left(1-v_{1}^{2}\right) E_{2}}
$$

where $E_{1}$ and $E_{2}$ are Young's modules and $v_{1}$ and $v_{1}$ are Poisson's ratios for the surfaces.

The contact was assumed fully flooded with the lubrication flow modelled with the Reynolds equation for thin films [52]. The differential equation was solved as the average in the height direction since the height of the lubrication film is much smaller than both the width and the length of the contact. Typically the height of the lubrication film is less than a few micrometers while the contact width is at least two orders of magnitude greater. The interaction between contact surfaces and the lubrication was modelled with no slip boundary conditions giving the fluid in contact with the wall the same velocity as the wall. To capture the effects of the asperity a 3 dimensional simulation was needed. The length axis of the cylinder was oriented along the $y$-axis yielding rolling along the $x$-axis in Fig. 2b. The Reynolds equation can then be formulated

$$
\frac{\partial}{\partial x}\left(\frac{\rho h^{3}}{12 \eta} \frac{\partial}{\partial x} p\right)+\frac{\partial}{\partial y}\left(\frac{\rho h^{3}}{12 \eta} \frac{\partial}{\partial y} p\right)-u_{\mathrm{m}} \frac{\partial}{\partial x}(\rho h)-\frac{\partial}{\partial t}(\rho h)=0
$$

The lubricants can often not sustain negative pressures. Instead lubricant cavitation limits the pressure from becoming negative, which was accounted for by forcing the pressure to be zero or positive.

Rolling contact fatigue typically appears after running in of the gears. After the running in process with surface hardening, asperity flattening by wear and plastic deformation, the surfaces will only deform elastically [11] [53] [54] [55]. By assuming purely elastic deformation the shape of the film thickness was described by

$$
h(x, y)=h_{0}-a_{\mathrm{sh}}(x, y, t)+\frac{x^{2}}{2 r_{\mathrm{x}}}+\frac{2}{\pi E^{\prime}} \int_{-\infty-\infty}^{\infty} \int_{-\infty}^{\infty} \frac{p d x^{\prime} d y^{\prime}}{\sqrt{\left(x-x^{\prime}\right)\left(y-y^{\prime}\right)}}
$$

The last term in Eq. (4) represents the elastic deformation of the two bodies and was derived from the Boussinesq potential functions [56]. The lubrication offset height $h_{0}$ was determined through the requirement of load balance of the contact,

$$
\int_{-\infty}^{\infty} \int_{-\infty}^{\infty} p d x d y-f=0
$$

where $f$ is the applied normal contact load. The normal contact load gives the Hertzian contact half width $a$ and the maximum pressure $p_{\text {Hertz }}[56]$ 


$$
\begin{aligned}
& a=\sqrt{\frac{8 f r_{x}}{\pi E^{\prime}}} \\
& p_{\text {Hertz }}=\frac{2 f}{\pi a}
\end{aligned}
$$

for the cylinder contact.

The properties of the lubrication film were modelled according to the formulation by Larsson et al. [57] of Dowson and Higginson's relation and Roelands equation [18] [58]. The pressure density relationship was

$$
\rho=\rho_{0}\left[1+\frac{A_{1} p}{1+A_{2} p}\right]\left[1-\alpha\left(\Gamma-\Gamma_{40}\right)\right]
$$

where $\rho_{0}$ is the density at the reference temperature $\Gamma_{40}=40{ }^{\circ} \mathrm{C}$. The thermal expansion variable $\alpha$ was modelled following Salehizadeh and Saka [59]

$$
\alpha=\alpha_{40} e^{-c p}
$$

where, $c$ is a constant equal to $1.51 / \mathrm{GPa}$ and $\alpha_{40}$ is the thermal expansion coefficient at the reference temperature $\Gamma_{40}=40{ }^{\circ} \mathrm{C}$. The pressure viscosity relationship was described by the Roelands equation [58]

$$
\eta=\eta_{0}(\Gamma) \exp \left(\left[\ln \left(\eta_{0}(\Gamma)\right)+9.67\right]\left[-1+\left(1+5.1 \cdot 10^{-9} p\right)^{Z_{\mathrm{R}}(\Gamma)}\right]\right)
$$

where the temperature exponent $Z_{\mathrm{R}}(\Gamma)$ was defined as

$$
Z_{\mathrm{R}}(\Gamma)=D_{\mathrm{Z}}+C_{\mathrm{Z}} \log \left(1+\frac{\Gamma}{135}\right)
$$

and the dynamic viscosity $\eta_{0}$ at atmospheric pressure was obtained from

$$
\log \left(\eta_{0}(\Gamma)\right)=-4.2+G_{0}\left(1+\frac{\Gamma}{135}\right)^{-S_{0}}
$$

Values for the coefficients $C_{\mathrm{z}}, D_{\mathrm{z}}, G_{o}$ and $S_{o}$ are given by Larsson et al. [57] for some common lubricants.

Since the analysis focused on the contact at the pitch line, the contact was modelled in pure rolling with the cylinder and the flat surface moving at the same velocity, $u_{\mathrm{m}}$, the only flow component which will give rise to shear stresses on the surfaces was the Poiseuille term in Eq. (3). If a sliding speed, $u_{\mathrm{s}} \neq 0$, had been introduced, then the Couette term would give further shear stresses. Based on the assumption of Newtonian flow and the no slip boundary condition, the shear stresses on the top or curved surface in Fig. 2b was evaluated by [6o] 


$$
\begin{aligned}
\tau_{x z} & =\frac{h}{2} \frac{\partial p}{\partial x}+\eta \frac{u_{\mathrm{s}}}{h} \\
\tau_{y z} & =\frac{h}{2} \frac{\partial p}{\partial y}
\end{aligned}
$$

The flat bottom surface was subjected to shear stresses of the same magnitude but with the opposite sign for the Poiseuille term. A similar set-up was used by among others Li and Kahraman [61].

The asperity was placed on the cylinder surface. The asperity shape $a_{\mathrm{sh}}$ in Eq. (4) was modelled in accord to Hannes and Alfredsson [7] with one cosine cycle

$$
a_{\mathrm{sh}}(x, y, t)=\frac{\delta}{2}\left(1+\cos \left[2 \pi \sqrt{\left(\frac{x-x_{\mathrm{d}}(t)}{\omega_{x}}\right)^{2}+\left(\frac{y}{\omega_{y}}\right)^{2}}\right]\right)
$$

in the region where

$$
\sqrt{\left(\frac{x-x_{\mathrm{d}}}{\omega_{x}}\right)^{2}+\left(\frac{y}{\omega_{y}}\right)^{2}}<\frac{1}{2}
$$

In Eqs (13) and (14) $\omega_{x}$ and $\omega_{y}$ are the asperity widths and $\delta$ is the asperity height, see Fig. $2 b$. For an elliptical asperity with no sharp edges, the widths are equal to the ellipse axes.

\subsection{Stresses and fatigue}

The surface stresses in the substrate were determined separately in a post processing step after solving the EHL equations. The stresses were computed using the Boussinesq, Cerruti and Flamant solutions in Appendix A. To reduce summation error and increase calculation speed the pressure and the shear traction in the rolling direction were divided into line loads and remaining point loads. The line loads were taken as the minimum values along each transverse line.

The Findley criterion was used to evaluate if the surface near the asperity was loaded above its fatigue limit [62]. In dimensionless form the Findley criterion is

$$
F i=\frac{\left(\tau_{\text {amp }}+\kappa \sigma_{\text {n,max }}\right)_{\max }}{\sigma_{\mathrm{eF}}}
$$

where $\kappa$ and $\sigma_{\mathrm{eF}}$ are material parameters, see Appendix B. The criterion was evaluated for all planes in each material point. Each plane was defined by its normal direction which was given by two angles $\theta$ and $\emptyset$ rotating to the right around the $z$ - and $y$-axis, respectively. Angular increments of $2^{\circ}$ were used here. A rotation matrix $\mathbf{R}=\mathbf{R}_{\varnothing} \mathbf{R}_{\theta}$ was used to rotate the stress tensor to the evaluated plane, $\mathbf{R} \boldsymbol{\sigma} \mathbf{R}^{\mathbf{T}}$. The shear stress amplitude, $\tau_{\mathrm{amp}}$, on the plane was combined with the maximum value of the normal stress, $\sigma_{\mathrm{n}, \max }$, found sometime during the 
load cycle on the plane. During the load cycle the shear stress vector described a loop on the plane. The shear stress amplitude on the plane was found as half of the distance between the two shear stress points that were the furthest apart. The critical plane was identified by the highest $F i$. If $F i$ is above unity for a material point, then the Findley criterion predicts that this point will not sustain infinite life. Socie and Marquis describe the criterion in more detail [62].

The parameters for the Findley criterion were determined from uni-axial data in the literature for the case carburized gear steel in Fig. 1. The procedure described in Appendix B gave $\kappa=$ 0.627 and $\sigma_{\mathrm{eF}}=625 \mathrm{MPa}$. A uni-axial bending fatigue stress $\sigma_{\mathrm{eb}}=692 \mathrm{MPa}$ was also determined, see Appendix B.

\section{Numerical model}

The pressure distributions in EHL contacts are determined numerically. Current models are often based on either finite element method (FEM), like the setup of Shirzadegan [63], or based on finite difference methods (FDM), like those described by Venner in [23] and by Huang in [64] and [65]. In particular, metal contact during the interaction of rough surfaces requires special numerical treatment. One method is to model dry micro-scale asperity contacts and superposition the contact pressure for the roughness on the EHL pressure while keeping the load balance [18]. Alternatively, Eq. (3) is solved for the rough surface profile with attention paid to nodes where the film thickness becomes smaller than a limiting value, which then represents metal contact. For such nodes the Poiseuille flow and the transient term in Eq. (3) become small and can be removed from the iterations for rapidly conversion to a stable solution [66]. Instead of removing these equation parts, the pressure on the nodes can be increased until the limit film thickness is reached locally. The pressure increase is given by Love's equation for elastic deflection in Eq. (4).

\subsection{Numerical setup}

The present numerical model was based on the FDM code by Huang [64] and incorporated the roughness within the EHL iterations by increasing the pressure on nodes with metal contact. The set-up utilised dimensionless parameters

$$
\begin{aligned}
& P=p / p_{\text {Hertz }}, \quad \bar{\rho}=\rho / \rho_{0}, \quad H=h r_{x} / a^{2} \\
& A_{\mathrm{sh}}=a_{\mathrm{sh}} r_{x} / a^{2}, X=x / a, \quad Y=y / a \\
& \Delta X=\Delta x / a, \quad \Delta Y=\Delta y / a \\
& \Omega_{x}=\omega_{x} / a, \quad \Omega_{y}=\omega_{y} / a, \quad \Delta=\delta r_{x} / a^{2} \\
& T=t u_{\mathrm{m}} / a, \quad \Delta T=u_{\mathrm{m}}\left(X_{\mathrm{e}}-X_{0}\right) /\left(N_{t} u_{\mathrm{c}}\right)
\end{aligned}
$$

To simplify Reynolds Eq. (3), $\lambda=\frac{12 u_{\mathrm{m}} r_{x}{ }^{2}}{a^{3} p_{\text {Hertz }}}$ and $\varepsilon=\frac{\bar{\rho} H^{3}}{\eta \lambda}$ were introduced. The space discretization of the Poiseuille flow term was performed with the approximation

$$
\left(\frac{\partial}{\partial X}\left(\varepsilon_{i, j} \frac{\partial}{\partial X} P_{i, j}\right)\right)=\frac{\left(\varepsilon_{i+1, j}+\varepsilon_{i, j}\right) P_{i+1, j}-\left(2 \varepsilon_{i, j}+\varepsilon_{i+1, j}+\varepsilon_{i-1, j}\right) P_{i, j}+\left(\varepsilon_{i, j}+\varepsilon_{i-1, j}\right) P_{i-1, j}}{2 \Delta X^{2}}
$$


of the second derivative, where the $i$ and $j$ refers to the node numbers in the $x$ - and $y$ directions. The second order approximation was also used for the Couette flow term

$$
\frac{\partial}{\partial X}\left(\bar{\rho}_{i, j} H_{i, j}\right)=\bar{\rho}_{i, j} \frac{3 H_{i, j}-4 H_{i-1, j}+H_{i-2, j}}{\Delta X}+H_{i, j} \frac{\partial \bar{\rho}_{i, j}}{\partial P_{i, j}} \frac{3 P_{i, j}-4 P_{i-1, j}+P_{i-2, j}}{\Delta X}
$$

The time dimension was discretized with the Crank-Nicolson method yielding the numerical setup of Reynolds equation

$$
\begin{aligned}
& \left(\frac{\partial}{\partial X}\left(\varepsilon_{i, j} \frac{\partial}{\partial X} P_{i, j}\right)\right)_{t_{n+1}}+\left(\frac{\partial}{\partial Y}\left(\varepsilon_{i, j} \frac{\partial}{\partial Y} P_{i, j}\right)\right)_{t_{n+1}}-\left(\frac{\partial \bar{\rho}_{i, j} H_{i, j}}{\partial X}\right)_{t_{n+1}} \\
& +\left(\frac{\partial}{\partial X}\left(\varepsilon_{i, j} \frac{\partial}{\partial X} P_{i, j}\right)\right)_{t_{n}}+\left(\frac{\partial}{\partial Y}\left(\varepsilon_{i, j} \frac{\partial}{\partial Y} P_{i, j}\right)\right)_{t_{n}}-\left(\frac{\partial \bar{\rho}_{i, j} H_{i, j}}{\partial X}\right)_{t_{n}} \\
& -2\left(\frac{\left(\bar{\rho}_{i, j} H_{i, j}\right)_{t_{n+1}}-\left(\bar{\rho}_{i, j} H_{i, j}\right)_{t_{n}}}{\Delta T}\right)=0
\end{aligned}
$$

where $t_{n}$ and $t_{n+1}$ indicate the current and next time step. In Eq. (19) the Poiseuille terms was evaluated according to Eq. (17) and the Couette flow terms according to Eq. (18).

To update the pressure, the residual of Eq. (19) was minimised using either the Gauss-Seidel or the Jacobi iteration methods in accord with Huang [64]. Like Huang, line relaxation was used to increase the convergence speed. At each iteration, the lubrication parameters and the lubrication film thickness in Eq. (19) were updated to fully couple the behaviour of the different physical properties.

The lubricant boundary conditions included zero contact pressure at the inlet, $X=X_{0}$, and the outlet, $X=X_{\mathrm{e}}$, see Fig. 3. On the transverse sides of the model, at $Y=-Y_{0}$ and $Y=0$, the pressure gradient $\partial P / \partial Y=0$ and thereby the lubrication flux was zero. The model width $Y_{0}$ was selected sufficiently large for the pressure profile at $Y=-Y_{0}$ to be undisturbed by the asperity. See Fig. 4a for cylindrical pressure profiles with and without a point asperity.

The pressure region required for determining the elastic displacements in Eqs (21) and (22) with good resolution was however wider than the relatively narrow model width $Y_{0}$ where the pressure and lubrication height were evaluated. For pressure positions outside the model width at $Y<-Y_{0}=-0.5\left(N_{y}-1\right) \Delta X$ and $Y>\mathrm{Y}_{0}=0.5\left(N_{y}-1\right) \Delta X$ the pressure $P$ was set equal to the cylindrical pressure at the border, $Y=Y_{0}$. 


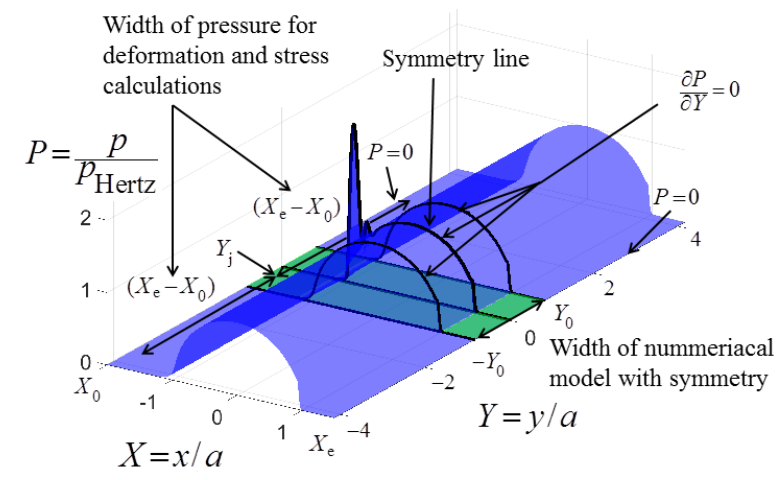

Fig. 3. Utilizing symmetry at $Y=0, P$ and $H$ were evaluated for $-Y_{0} \leq Y \leq 0$. The elastic deformation required the larger pressure domain $Y \leq\left|Y_{0}+X_{\mathrm{e}}-X_{0}\right|$.

The elastic surface displacements in Eq. (4) were evaluated numerically with Loves expressions for elastic deflection due to uniformed pressures applied to rectangular regions [67]. The displacement of each node $(i, j)$ was summed for all pressured regions in the $x$ direction at $X_{0} \leq X \leq X_{\mathrm{e}}$ in Fig. 3, and on all regions in the distance $X_{\mathrm{e}}-X_{0}$ in both the positive and the negative $y$-direction from $Y_{j}, Y_{j}-\left(X_{\mathrm{e}}-X_{0}\right) \leq Y \leq Y_{j}+\left(X_{\mathrm{e}}-X_{0}\right)$ in Fig. 3. The asperity shape function $A_{\text {sh }}$ in Eqs (4) and (21) depended on the asperity position

$$
X_{\mathrm{d}}=X_{0}+T \frac{u_{\mathrm{c}}}{u_{\mathrm{m}}}
$$

where $T=0$ at simulation start when $X_{\mathrm{d}}=X_{0}$. Hence, Eq. (4) was evaluated numerically as

$$
\begin{gathered}
H_{i, j}=H_{0}+X_{i}^{2}+\frac{2}{\pi E^{\prime}} \sum_{k=1}^{N_{x}} \sum_{l=j-N_{x}}^{j+N_{x}} P(k, l)\left(A_{\mathrm{D}}+B_{\mathrm{D}}+C_{\mathrm{D}}+D_{\mathrm{D}}\right)-A_{\mathrm{sh}}(i, j, t) \\
A_{\mathrm{D}}=\left(X^{\prime}+\Delta X\right) \ln \left[\frac{\left(Y^{\prime}+\Delta X\right)+\left\{\left(Y^{\prime}+\Delta X\right)^{2}+\left(X^{\prime}+\Delta X\right)^{2}\right\}^{0.5}}{\left(Y^{\prime}-\Delta X\right)+\left\{\left(Y^{\prime}-\Delta X\right)^{2}+\left(X^{\prime}+\Delta X\right)^{2}\right\}^{0.5}}\right] \\
B_{\mathrm{D}}=\left(X^{\prime}+\Delta X\right) \ln \left[\frac{\left(Y^{\prime}+\Delta X\right)+\left\{\left(Y^{\prime}+\Delta X\right)^{2}+\left(X^{\prime}+\Delta X\right)^{2}\right\}^{0.5}}{\left(Y^{\prime}-\Delta X\right)+\left\{\left(Y^{\prime}+\Delta X\right)^{2}+\left(X^{\prime}-\Delta X\right)^{2}\right\}^{0.5}}\right] \\
C_{\mathrm{D}}=\left(X^{\prime}-\Delta X\right) \ln \left[\frac{\left(Y^{\prime}-\Delta X\right)+\left\{\left(Y^{\prime}-\Delta X\right)^{2}+\left(X^{\prime}-\Delta X\right)^{2}\right\}^{0.5}}{\left(Y^{\prime}+\Delta X\right)+\left\{\left(Y^{\prime}+\Delta X\right)^{2}+\left(X^{\prime}-\Delta X\right)^{2}\right\}^{0.5}}\right] \\
D_{\mathrm{D}}=\left(X^{\prime}-\Delta X\right) \ln \left[\frac{\left(Y^{\prime}-\Delta X\right)+\left\{\left(Y^{\prime}-\Delta X\right)^{2}+\left(X^{\prime}-\Delta X\right)^{2}\right\}^{0.5}}{\left(Y^{\prime}+\Delta X\right)+\left\{\left(Y^{\prime}-\Delta X\right)^{2}+\left(X^{\prime}+\Delta X\right)^{2}\right\}^{0.5}}\right]
\end{gathered}
$$


where $X^{\prime}=X_{i}-X_{k}$ and $Y^{\prime}=Y_{j}-Y_{l}$. The deflections were the sum of the contributions from $N_{x}$ nodes in the $x$-direction and $2 N_{x}$ nodes in the $y$-directions.

Since the asperity was small compared to the cylindrical contact, it was assumed that the single asperity would not change the overall lubrication height or offset, $H_{0}$. The offset was obtained during the initial time independent simulation of a smooth rolling cylinder. The offset, $H_{0}$, was then kept throughout the time dependent simulation when the asperity was over-rolled. It was determined through Newton-Raphson iterations of the load balance in Eq. (5), where the pressure depended on the lubrication offset. To yield a stable procedure the lubrication offset was only updated once the total load difference between two iterations was less than $1 \%$ and either the sum over all nodes of the residual of Reynolds Eq. (19) was below 0.001 or 20 iterations had been performed.

Following $\mathrm{Zhu}$ and $\mathrm{Hu}$ [66], the dimensionless lubrication height on the asperity was restricted to be larger or equal to a minimum value which was assumed to be equivalent to solid contact. The condition was enforced by increasing the pressure during the iterations on each node where $H$ was lower than the specified limit. $\mathrm{Zhu}$ and $\mathrm{Hu}$ set the minimum value to $10^{-5}$. For this work, $5 \cdot 10^{-6}$ was used.

\subsection{Numerical simulation}

The numerical simulation started with solving for the time independent smooth rolling cylinder against a flat surface. In order to rapidly reach convergence for the pressure and lubrication offset, a mesh refinement procedure was used. When $P$ and $H_{0}$ had approximately converged for the coarse mesh, this solution was taken as the start value for the refined mesh. The refined mesh had double number of nodes in both $x$ - and $y$-directions. The mesh was refined two times this way to the final mesh with $201 \cdot 97$ nodes in the $x-y$ plane. When the time independent solution had converged for the final mesh, the time dependent solution was introduced. To achieve a stable transition from the time independent to the time dependent case, the time dependent terms where gradually introduced over 10 steps. Thereafter, the asperity was inserted at $X_{\mathrm{d}}=X_{0}$ and moved through the contact at $N_{t}$ number of time steps. To stabilise the procedure, limits were used for the pressure increments. The pressure increment on an individual node was not allowed to be higher than 0.02 or lower than -0.1 . If contact was detected, then pressure increments up to 0.2 were allowed. The transverse symmetry was utilized, which reduced the number of node lines in the $y$-directions with numerical solution to 49. The solution for the remaining 48 lines was obtained by mirroring the values around the symmetry line in the $\mathrm{RD}$, see Fig. 3. Appendix $\mathrm{C}$ includes a model validation against results by Holmes et al. [68].

The number of nodes and time steps was a compromise between solution time and accuracy. Holmes et al. [68] investigated the influence of spatial and time resolution for the transient problem of a spherical contact over-rolling a transvers ridge. The accuracy of the present model was evaluated similarly; see Appendix C. Based on the analyses, the selected space resolution with $201 \cdot 97$ nodes in the $x-y$ directions together with 402 time steps for the overrolling sequence was judged as a sufficiently good compromise between accuracy and simulation time. With this resolution, the transient simulation of over-rolling the asperity required about $120 \mathrm{CPU}$ hours on the available computer cluster. Holmes et al. also considered the performance of different discretization techniques and found that the presently used Crank-Nicolson time implementation performed the best.

For simulations with other $p_{\text {Hertz }}$ than the reference value in Table 1 the contact half width changed. However, the investigated asperity size remained fixed. In order to keep the fatigue 
resolution at the asperity comparable between different $p$, the spatial distances between nodes $\Delta x, \Delta y$ were fixed. Therefore, the dimensionless variables $X, Y$ and boundaries, $X_{\mathrm{o}}, X_{\mathrm{e}}, Y_{\mathrm{o}}$, were rescaled based on the ratio between the current and the reference contact half width. The rescaling ensured that the spatial distance between nodes, the size of the numerical model and the number of nodes was constant regardless of $p_{\text {Hertz }}$. However, the number of nodes per contact half width changed for the different $p_{\text {Hertz }}$.

\section{Results}

Table 1 presents mechanical and numerical parameters for the analysed gear contact in Fig. 1a. The asperity data in Table 1 were taken from Dahlberg and Alfredsson [6] who measured the surface roughness on the gear teeth. The material followed Swedish standard SS142506 carburized and heat treated to $750-800 \mathrm{HV}$ [69]. The investigation contained effects of the asperity geometry, maximum Hertzian contact pressure, temperature and lubrication on the surface stresses. The asperity geometry was varied in size and shape. The circular shape was changed to an ellipse aligned with the rolling or the transverse direction. The asperity was also replaced with a smooth and circular indent. Throughout the analysis the cylinder radius, velocity and cylinder material remained unchanged.

The lubrication parameters in Table 2 for the pressure-viscosity and the pressure-density relations were taken from Larsson [57] and are representative for gears. PAO B was selected as the reference lubricant since it is similar to the lubrication used in the analysed gear contact. When nothing else is stated, PAO B at $90{ }^{\circ} \mathrm{C}$ was used. Table 2 includes the dimensionless parameters

$$
\begin{aligned}
& W=\frac{f}{E^{\prime} r^{\prime}} \\
& G=\alpha_{\text {Barus }} E^{\prime} \\
& U=\frac{\eta_{0} u_{\mathrm{m}}}{E^{\prime} r^{\prime}}
\end{aligned}
$$

evaluated for the conditions of Table 1, with $\alpha_{\text {Barus }}$ evaluated at atmospheric pressure. These parameters were included for comparison to EHL simulations in the literature. 
Table 1. Mechanical and numerical parameters including the reference asperity.

\begin{tabular}{lccc}
\hline Parameter & Symbol & Value & Unit \\
\hline Speed of curved surface & $u_{\mathrm{c}}$ & 8.5 & $\mathrm{~m} / \mathrm{s}$ \\
Speed of flat surface & $u_{\mathrm{f}}$ & 8.5 & $\mathrm{~m} / \mathrm{s}$ \\
Slide to Roll Ratio & $S R R$ & 0 & - \\
Hertz pressure & $p_{\mathrm{Hertz}}$ & 1.93 & $\mathrm{GPa}$ \\
Equivalent elastic modulus & $E^{\prime}$ & 226 & $\mathrm{GPa}$ \\
Equivalent radius & $r^{\prime}$ & 10.6 & $\mathrm{~mm}$ \\
Contact half width, Eq. (6) & $a$ & 362 & $\mu \mathrm{m}$ \\
Asperity height & $\delta$ & 1.5 & $\mu \mathrm{m}$ \\
Asperity wavelength, $x$ direction & $\omega_{x}$ & 100 & $\mu \mathrm{m}$ \\
Asperity wavelength, $y$ direction & $\omega_{y}$ & 100 & $\mu \mathrm{m}$ \\
Temperature & $\Gamma$ & 90 & ${ }^{\circ} \mathrm{C}$ \\
Inlet position & $X_{0}$ & -2.0 & - \\
Outlet position & $X_{\mathrm{e}}$ & 1.5 & - \\
Transvers width & $Y_{\mathrm{O}}$ & 0.66 & - \\
Number of nodes & $N_{X} \cdot N_{Y}$ & $257 \cdot 97$ & - \\
Number of time steps & $N_{t}$ & 514 & - \\
\hline
\end{tabular}

Table 2. Lubricant data, $\eta_{o}$ and $\alpha_{\text {Barus }}$ evaluated for $p=1$ atm and $Z, W, G$ and $U$ for the conditions in Table 1.

\begin{tabular}{lcccccc}
\hline & $\begin{array}{c}\text { Paraffinic } \\
\text { mineral }\end{array}$ & PAO B & PAO A & $\begin{array}{c}\text { Naphthenic } \\
\text { paraffinic } \\
\text { VG150 }\end{array}$ & $\begin{array}{c}\text { Naphthenic } \\
\text { paraffinic } \\
\text { VG68 }\end{array}$ & $\begin{array}{c}\text { Naphthenic } \\
\text { paraffinic } \\
\text { VG32 }\end{array}$ \\
\hline$S_{0} /-$ & 1.31 & 1.25 & 1.37 & 1.50 & 1.68 & 1.58 \\
$G_{0} /-$ & 4.76 & 4.57 & 4.62 & 4.96 & 4.49 & 3.98 \\
$C_{\mathrm{z}} /-$ & 0.229 & -0.0710 & 0.275 & -0.443 & 0.010 & -0.014 \\
$D_{\mathrm{z}} /-$ & 0.541 & 0.500 & 0.447 & 0.711 & 0.692 & 0.727 \\
$\eta_{0} /(\mathrm{mPa} \cdot \mathrm{s})$ & 17 & 16 & 12 & 13 & 5.1 & 3.8 \\
$\alpha_{\mathrm{Barus}} / \mathrm{GPa}^{-1}$ & 17 & 14 & 14 & 17 & 16 & 15 \\
$Z_{\mathrm{R}} /-$ & 0.59 & 0.48 & 0.51 & 0.61 & 0.69 & 0.72 \\
$A_{1} / \mathrm{GPa}^{-1}$ & 0.640 & 0.690 & 0.690 & 0.630 & 0.630 & 0.630 \\
$A_{2} / \mathrm{GPa}^{-1}$ & 2.89 & 2.55 & 2.55 & 2.51 & 2.51 & 2.51 \\
$\alpha_{40} /\left(10^{-4}{ }^{\circ} \mathrm{C}\right)$ & 6.6 & 6.8 & 7.1 & 6.7 & 6.9 & 7.2 \\
$W / 10^{-4}$ & 4.6 & 4.6 & 4.6 & 4.6 & 4.6 & 4.6 \\
$G / 10^{3}$ & 3.8 & 3.1 & 3.1 & 3.8 & 3.5 & 3.4 \\
$U / 10^{-11}$ & 6.1 & 5.8 & 4.4 & 4.5 & 1.8 & 1.3 \\
\hline
\end{tabular}

\subsection{Asperities}

The pressure and lubrication film thickness are presented in Fig. 4a for the circular asperity contact in Table 1. The thick solid lines represent the time independent solution or smooth cylinder contact while the thin lines represent the time dependent solution along $Y=0$ with the asperity at three instances or positions during the over-rolling. The thin solid lines illustrate the conditions at $X_{\mathrm{d}}=-0.9$ or just after the asperity entered into the contact. The pressure spike on the asperity was already well developed and in front of the asperity the squeeze effect has created a small pressure peak. Just in front of and behind the asperity the contact pressure was slightly relieved compared to the cylindrical pressure, which agree with 
the findings by Deolalikar et al. [41]. The film thickness tended to zero at the asperity centre. The dashed lines presents the conditions with the asperity at the contact centre at $X_{\mathrm{d}}=0.0$, and for the dash-dot lines the asperity is at the contact exit, $X_{\mathrm{d}}=0.9$. The curves show how the local contact properties developed at asperity entry and travelled with the asperity throughout the contact.

The pressure spike on the asperity in Fig. 4a is local and of point type. When assessing fatigue the first issue is whether there exist any tensile stresses in the surface during the over-rolling. Therefore, the first or major principle stress was captured for all locations and time instances during the over-rolling. Fig. $4 \mathrm{~b}$ shows a surface view of the contact with the maximum values of the major principal stress in each surface position sometime during the over-roll sequence. Note that the values at the different $x$ positions in the Fig. $4 \mathrm{~b}$ are from different time instances. There were two areas where positive stresses appeared in the surface. One was on the left side of the asperity as it entered the contact and the other was on its right side as it exited the contact. For the presently investigated pure rolling, the highest positive surface stresses occurred when the asperity exited the contact region. Fig. $4 \mathrm{~b}$ shows the full width of the numerical model between $\pm y_{0}= \pm 242 \mathrm{~mm}$. At the top and bottom edge of the figure the stress profile agrees with the contact pressure profile with $\sigma_{1}=\sigma_{y}$ from Appendix A. This shows that the model width was sufficiently large for the contact conditions to return to those of the cylindrical contact beside the asperity path.

Fig. 4c presents the major principal stress $\sigma_{1}$ in the area surrounding the asperity. Note that the contour values in this plot are the maximum over all time instances during the over rolling. The top part of Fig. 4c illustrates that the maximum normal stress was substantially tensile for material points on the asperity. In fact, the highest stresses were reached a small distance on the asperity sides in the transverse direction from the symmetry line. These highest values arose when the asperity exited the contact where it was loaded from a small angle yielding an elliptical contact surface. The region with high tensile $\sigma_{1}$ then surrounded the asperity peak on the contact outside. Slightly higher stress levels developed at asperity exit than at entry due to the asperity interaction with the asymmetric EHL pressure from pure rolling.

The directions of $\sigma_{1}$ are indicated with arrows in the bottom part of Fig. 4c. Note that the arrows point in both directions of the principal stress. They were only included for material point subjected to tensile $\sigma_{1}>200 \mathrm{MPa}$. The arrows are the projection of the direction on the $x-y$ plane, yielding short arrows for directions with large out of plane components.

Fig. $4 \mathrm{~d}$ presents the accumulated fatigue risk from the complete load cycle. The top part of the figure contains a contour plot of the Findley criterion in Eq. (15) at material points close to the asperity. The highest fatigue risk was reached at the positions that correspond to the tensile stress maxima in Fig. 4c. The positions were on the transverse sides of the asperity, about halfway between the peak and the transition to the smooth cylinder surface. The bottom part of Fig. 4d shows arrows which indicate the normal direction of the predicted critical plane.

Fig. 4a shows the asperity contact conditions at three instances. These contact views are important for determining properties related to the contact and lubrication conditions. Similarly, the contact view in Fig. 4b shows where in the contact the highest stresses developed. However, the graphs show the conditions at different material points which is of less importance for fatigue evaluation. Fatigue may develop due to the stress cycle in highly stressed material areas and therefore the stress conditions and stress cycle at such specific areas were investigated and presented in Fig. 4c and 4d. These graphs were here denoted 
material views in contrast to the contact views in Fig. 4a and $4 \mathrm{~b}$. The material views are presented using the moving coordinate system $x_{\mathrm{a}}-y_{\mathrm{a}}$ with origin at the asperity centre in Fig. $2 \mathrm{~b}$ whereas the contact views relate to the contact fixed coordinate system $x-y$.

Fig. 4a includes a dotted line which indicates the maximum asperity pressure for each $x$ position. The maximum was taken for all time instances during over rolling and all transverse $y$-coordinates at the $x$-position. At the inlet and outlet, the pressure peak on the asperity was slightly higher a short transverse distance from the symmetry line than on it. Therefore, the dotted line is slightly above the solid thin pressure line at $X_{\mathrm{d}}=-0.9$.
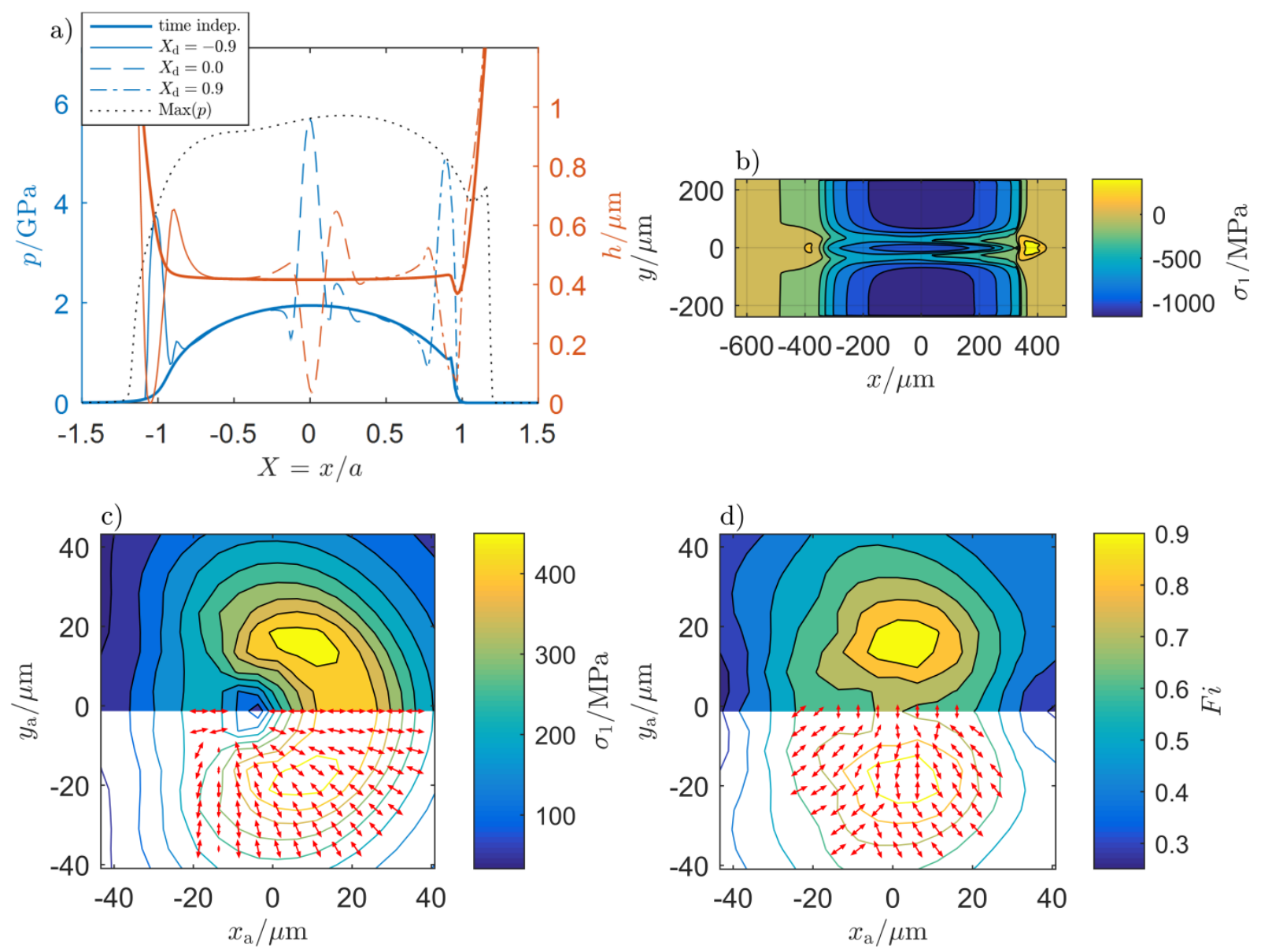

Fig. 4. Contact defined in Table 1 and Table 2: a) Contact view of $p$ and $h$. b) Maximum $\sigma_{1}$ during over-rolling. c) Value and direction of maximum $\sigma_{1}$ at points close to the asperity. d) Findley index and normal to the critical plane. RD is to the left.

The lubrication influences are presented in Fig. 5a to 5e for four of the oils in Table 2. Fig. 5a exemplifies the maximum pressure over time and $y$-positions. The figure shows that changing the lubricant may give noticeable influence on the asperity pressure. The dashed line for PAO $\mathrm{B}$ is also the dotted line in Fig. 4a. The two excluded lubricants in Table 2 were PAO A and Naphthenic VG68. The results for PAO A were marginally higher than PAO B and those for Naphthenic VG68 were between those for Naphthenic VG32 and VG150, see Fig. 5 f.

Fig. 5b presents the maximum $\sigma_{1}$ for each contact position. The maximum was for all time instances and all $y$-positions. The highest tensile stresses were reached at the exit side of the contact for all lubricants. However, Naphthenic VG32 displays almost as high $\sigma_{1}$ at the contact 
entry side, since its viscosity was so low that the load case was very similar to the dry contact case. In the dry contact case the stress levels would be symmetric around the contact centre line. The central film thickness for Naphthenic VG32 was $0.09 \mu \mathrm{m}$. Fig. 5a and b also include the smooth contact with lubrication PAO B as reference.

Fig. $5 \mathrm{c}, 5 \mathrm{~d}$ and $5 \mathrm{e}$ present maxima of $p, \sigma_{1}$ and $F i$ from a material point of view, i.e. for the $x_{\mathrm{a}}{ }^{-}$ coordinate at the asperity. Notice that the peak values in Fig. $5 \mathrm{~d}$ and $4 \mathrm{e}$ were located slightly to the right of the symmetry line in the same way as is visible in Fig. $4 \mathrm{c}$ and $4 \mathrm{~d}$. In front or to the right of the moving asperity in Fig. $5 c$ there are small $p$ peaks visible from the squeeze effect. The figures include the asperity profile $a_{\text {sh }}$ from Eq. (13) for identification of stress positions compared to the asperity.

Fig. 5 f compares the respective maximum of $p, \sigma_{1}$ and $F i$, denoted $\hat{p} \hat{\sigma}_{1} \hat{F} i$, during the asperity contact. The pressure was normalized with the maximum asperity pressure, $p_{\mathrm{dry}}=7.4 \mathrm{GPa}$, from the dry contact study of similar conditions [7]. The $\sigma_{1}$ was normalized with $\sigma_{\mathrm{eb}}=692$ MPa from Appendix B. The contacts with lower viscosity lubrications showed higher risk of fatigue failure. This agrees well with for instance Sharif et al. statement that lower ratio between lubrication height and roughness, which follows from lower viscosity, will yield higher fatigue risk [48].
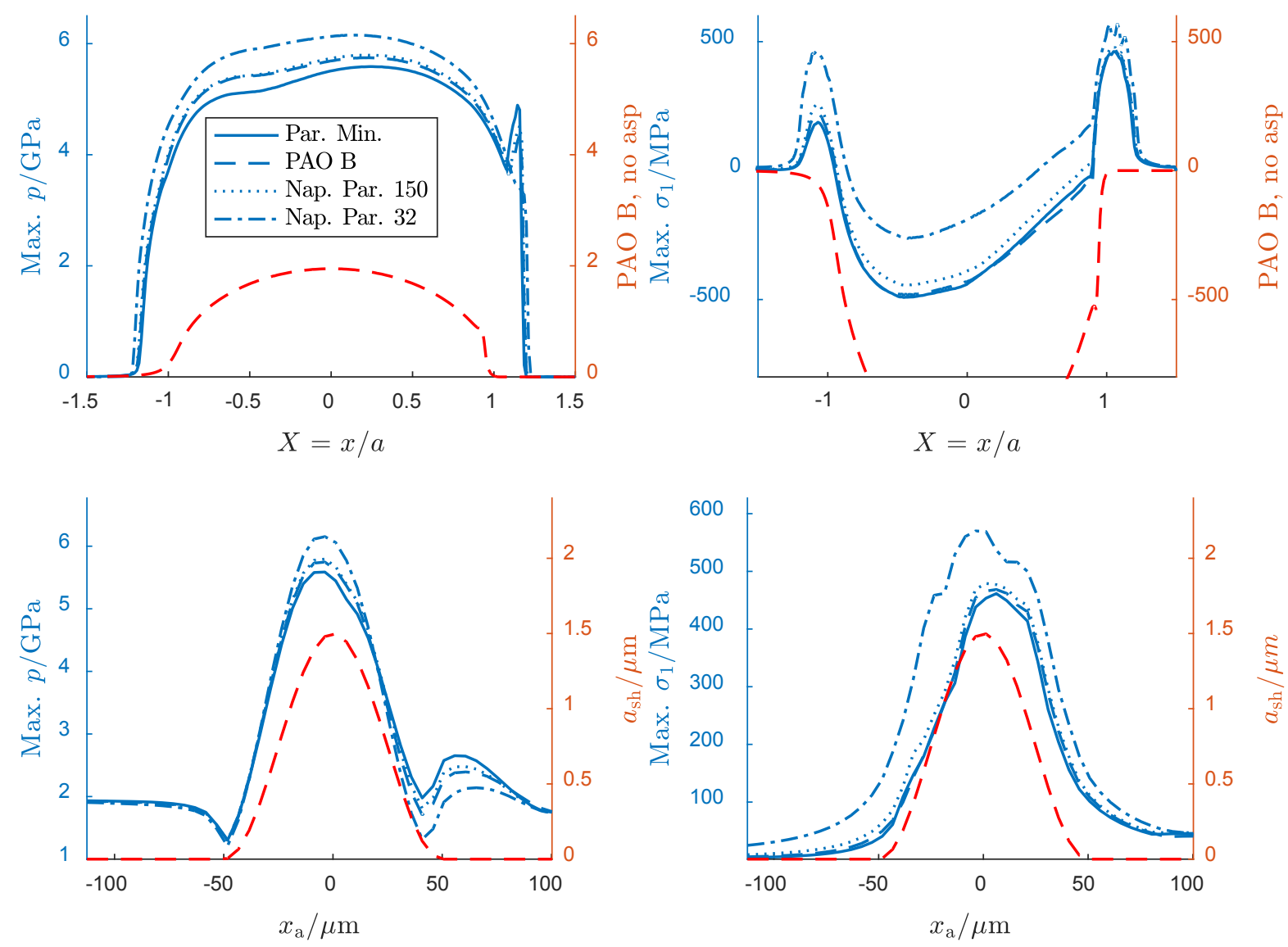

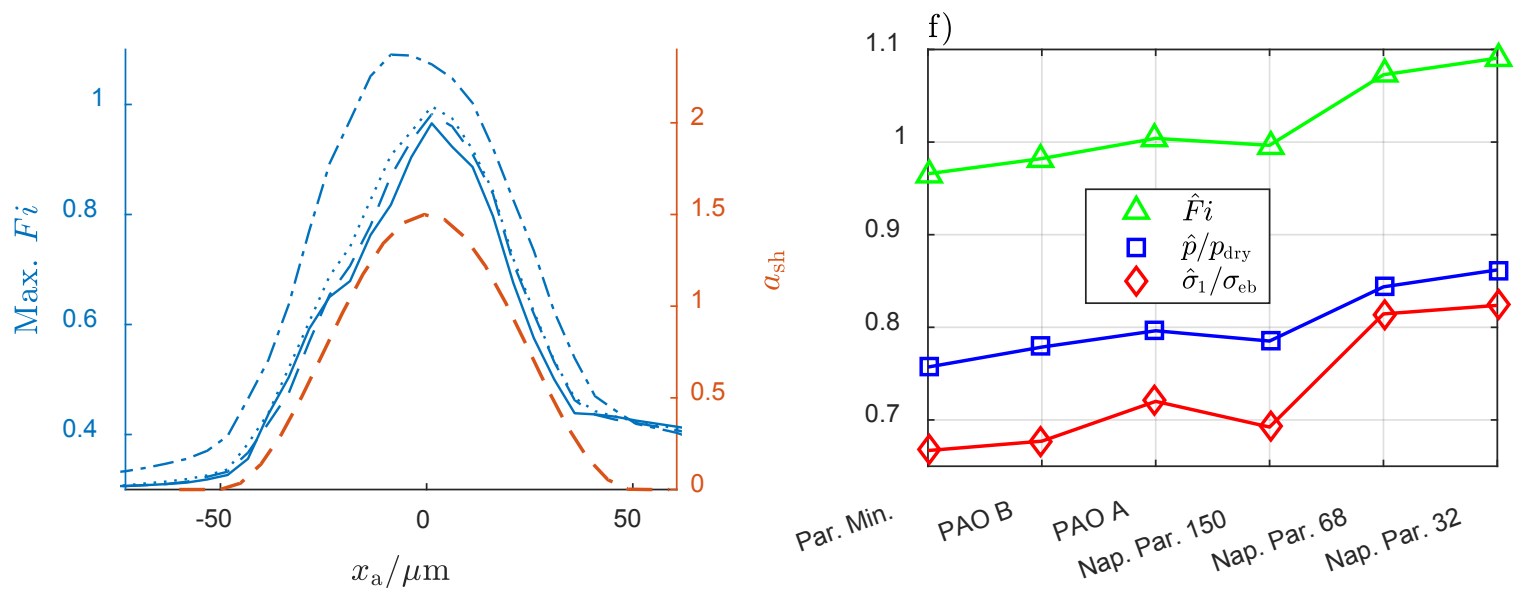

Fig. 5. The effect of different lubrications on pressure and surface stresses. a) - e) Maximum values over time and $y$-direction for each row of $x_{\mathrm{a}}$-coordinates. f) Maximum values for asperity contact cycle.

The effects of contact temperature are presented in Fig. $6, \Gamma=70-110{ }^{\circ} \mathrm{C}$. Fig. 6 a presents the largest Findley index for each $x_{\mathrm{a}}$. With increased temperature the viscosity decreased which reduced the film thickness and increased $\hat{p}$. In Fig. $6 \mathrm{~b}, \hat{p}$ will level off towards the dry contact pressure $p_{\text {dry }}$.
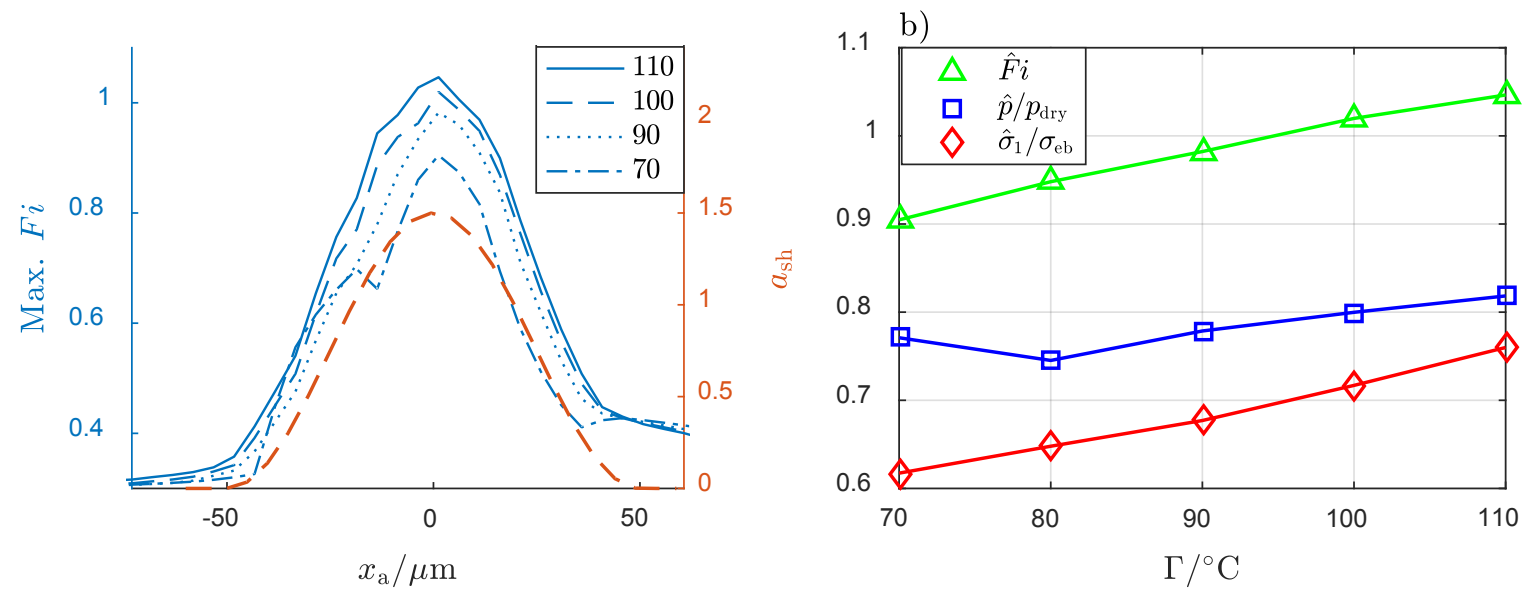

Fig. 6. Effects of contact temperature: a) Fatigue index, maximum over $y$. b) Maximum of $p$, $\sigma_{1}$ and $\mathrm{Fi}$.

Some effects of varying $p_{\text {Hertz }}$ from 0.4 to $2.4 \mathrm{GPa}$ are illustrated in Fig. 7. Fig. 7a shows two curves. One is the maximum asperity pressure during over rolling, $\hat{p}$. The other is the pressure increase on the asperity from $p_{\text {Hertz. }}$. The pressure increase was a not affected much by $p_{\text {Hertz, }}$ instead it stayed around a 3.5-4 GPa increase. Fig. $7 \mathrm{~b}$ shows how the fatigue risk at the asperity increases linearly with $p_{\text {Hertz. }}$. 

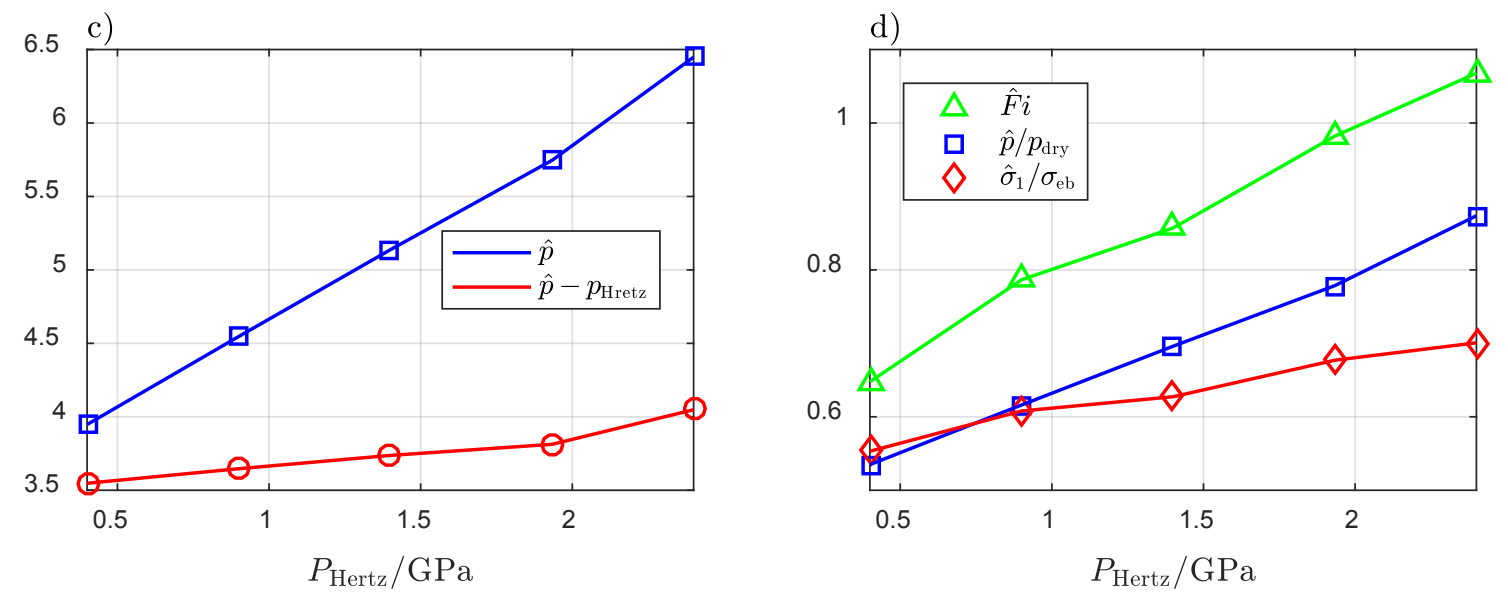

Fig. 7. Effect of $p_{\text {Hertz }}$ : a) Maximum $\hat{p}$ and increase $\hat{p}-p_{\text {Hertz }}$ on the asperity during overrolling. b) Summary of effects for $p_{\text {Hertz }}$.

Some geometrical properties of the asperity were investigated. The asperity height had an almost linear effect on the fatigue index in Fig. $8 \mathrm{a}$ and $7 \mathrm{~b}$. For all $\delta$, the point with the highest fatigue index was just to the right of the asperity centre. Fig. $8 \mathrm{c}$ and $7 \mathrm{~d}$ show the effect of different asperity widths, at $\delta=1.5 \mu \mathrm{m}$ from Table 1 . The narrow asperity display more concentrated and higher $\sigma_{\mathrm{eF}}$ than the others. The highest index was positioned just to the left of the asperity centre. For the wider asperities the location of the maximum index shifted to the right side. The largest index was located slightly off the centre line for all asperities. In Fig. $8 \mathrm{~d}$ the fatigue risk decreases exponentially with increasing asperity diameter. Also, for the narrowest asperity all normalized parameters are $>1$. Contact fatigue was therefore judged as possible for narrow asperities. 

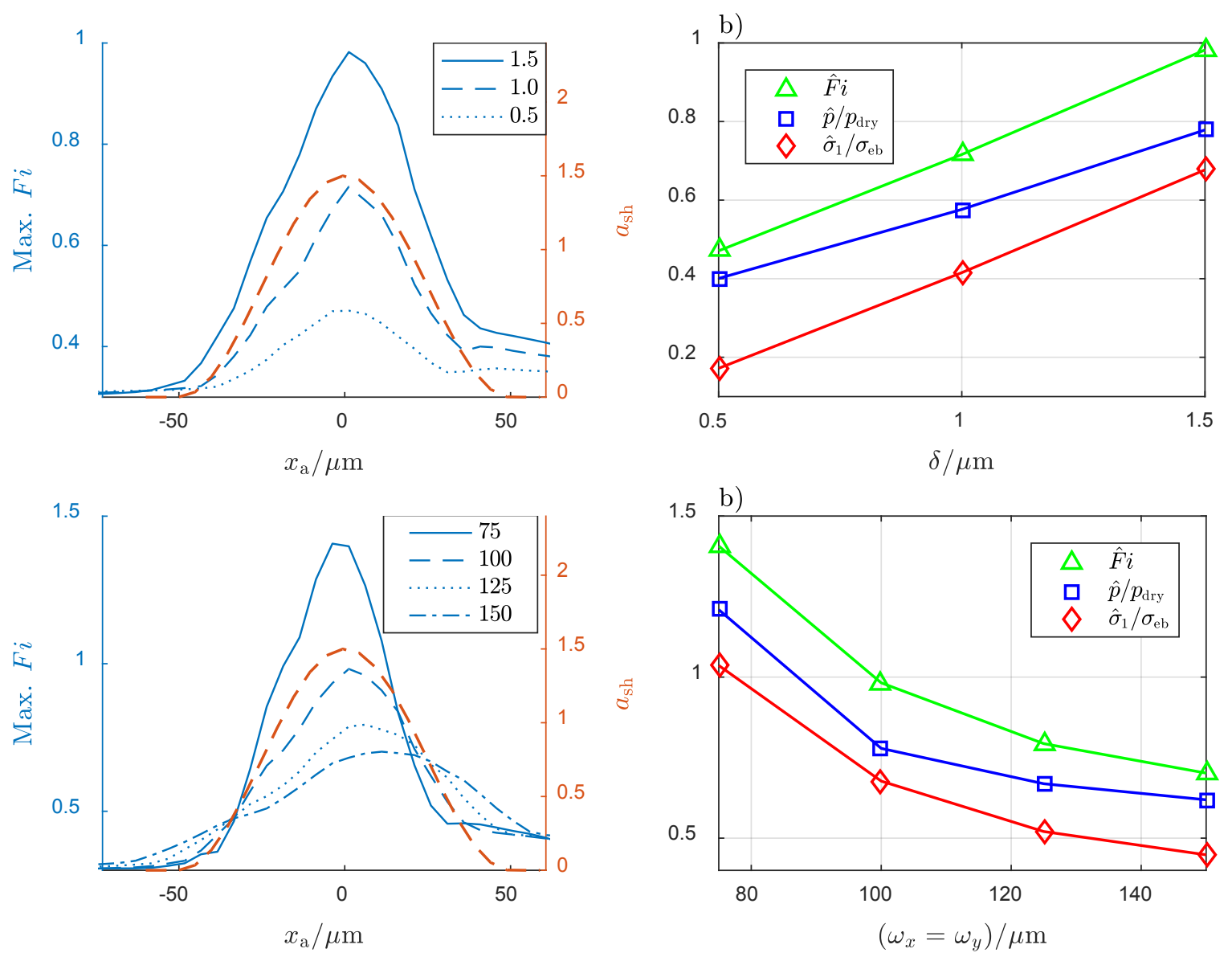

Fig. 8. Effects of asperity height $\delta$ in a) and b) for $\omega=100 \mu \mathrm{m}$. Effect of asperity width $\omega$ in c) and d) for $\delta=1.5 \mu \mathrm{m}$. Other data is in Table 1 and 2.

The asperity aspect ratio, expressed as $\omega_{x} / \omega_{y}$, was also considered, where $\omega_{y}$ is the asperity width or axis in the transverse $y$-direction and $\omega_{x}$ is the width in the rolling $x$-direction. In Fig. 9 the minor axis was always set to $100 \mu \mathrm{m}$. The dotted line illustrates the reference asperity also visible in Fig. 3. The summary results in Fig. 9b suggests fatigue for the circular asperity with $\omega_{x} / \omega_{y}=1$. The decrease in risk from this value was believed to be an effect of the constant minor axis and therefore comparing to larger asperities for $\omega_{x} \neq \omega_{y}$. For the asperities with $\omega_{x}>100 \mu \mathrm{m}$ the highest point of fatigue index was about 20-30 $\mu \mathrm{m}$ to the right while for $\omega_{x}<100$, the highest point of fatigue damage was at, or just to the left, of the asperity centre. 

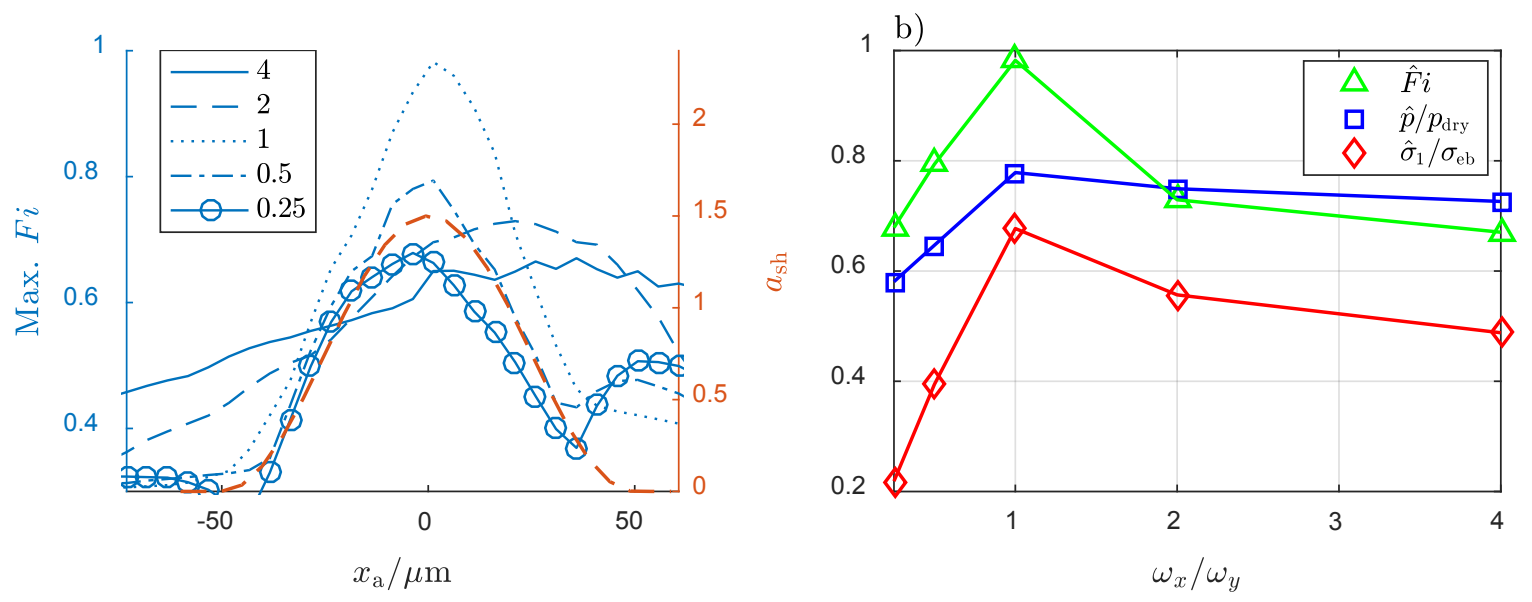

Fig. 9. Effects of elliptical asperities with aspect ratio $\omega_{x} / \omega_{y} \neq 1$ The minor axis was $100 \mu \mathrm{m}$. a) Findley index at asperity and b) summary of aspect ratio effects on fatigue.

\subsection{Dents}

The investigation included smooth sine shaped dents with negative $\delta<0$ in Eq. (13). The pressures and stresses around a dent with $\delta=-1.5 \mu \mathrm{m}$ are presented in Fig. 10 for comparison with the asperity in Fig. 4. Fig. 10a presents the pressure and film thickness. The time independent case has thick solid lines and the time dependent cases at $X_{\mathrm{d}}=-0.9 ; 0 ; 0.9$ have thin lines. The dashed lines illustrates a pressure dip to the right and at the centre of the dent, similar to the pressure increase to the right and on top of the asperity in Fig. 4a.

The maximum values of $\sigma_{1}, p$ and $F i$ were lower for a dent than for the corresponding asperity. The highest $\sigma_{1}$ on the symmetry line was here reached in the $y$-direction, see Fig. $10 \mathrm{c}$, compared to the $x$-direction for the asperity. The asperity generated a point load around which the tensile stresses arose. On the dent the pressure was instead relived, which opened for positive stresses inside the dent. Since the left rim of the dent was more heavily loaded than the right, the left side experienced higher stress levels. The highest fatigue index in Fig. 1od occurred on the symmetry lines and on the left side of the dent. 

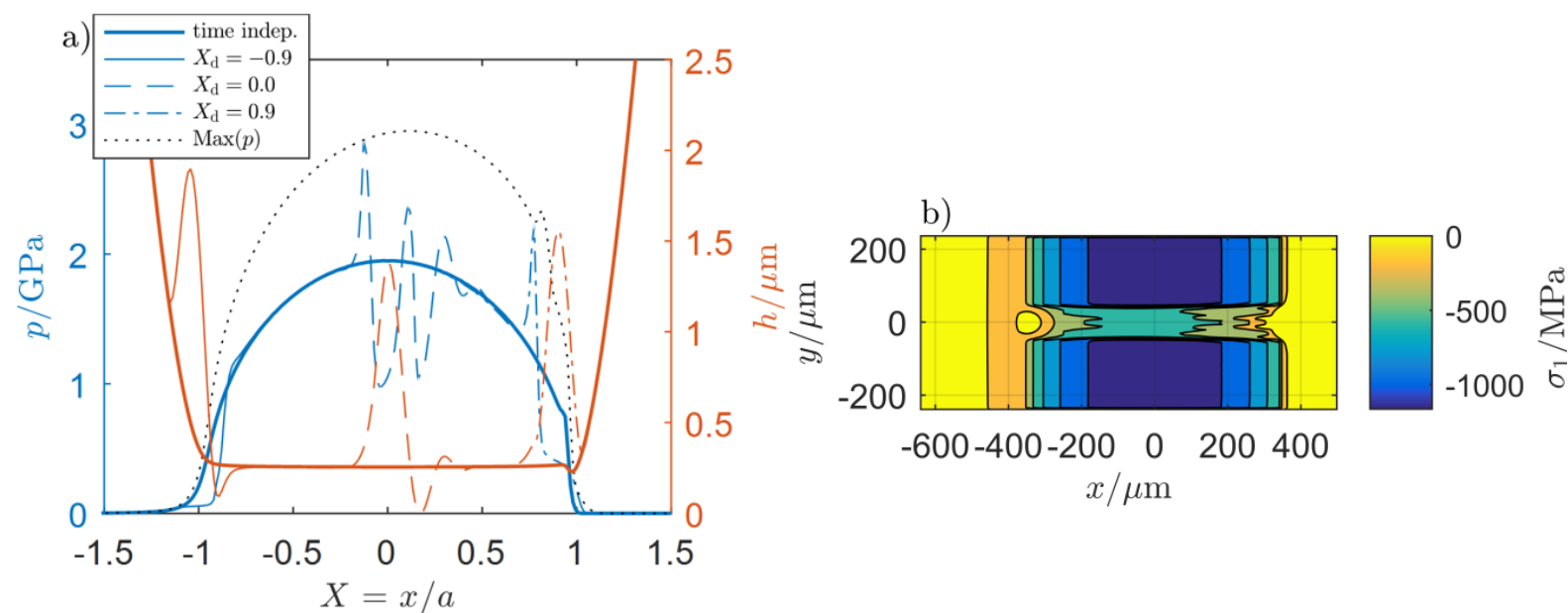

c)
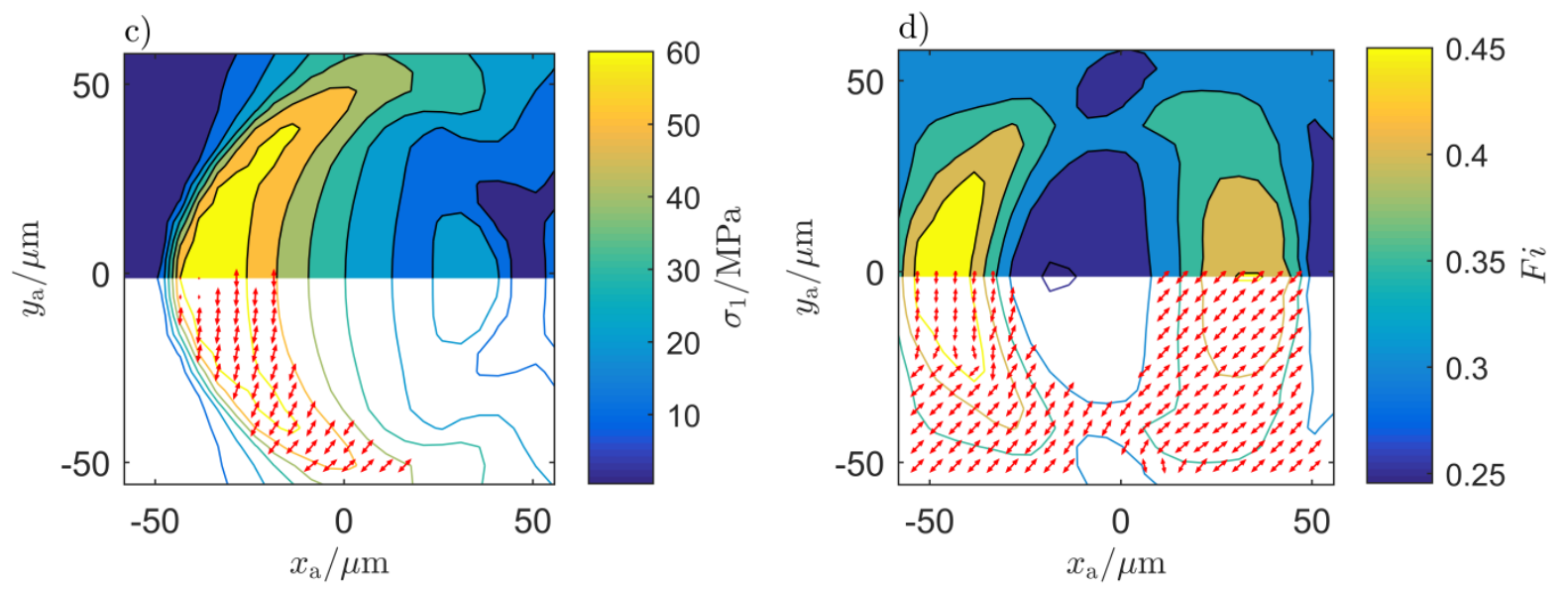

Fig. 10. Contact cycle for dent with $\delta=-1.5 \mathrm{~mm}$ and other data from Table 1. a) Contact view of $p$ and $h$. b) Maximum $\sigma_{1}$ during over-rolling. c) Maximum $\sigma_{1}$ and direction close to the dent. d) Findley index and normal to the critical plane. RD is to the left.

Fig. 11 shows the influences of $p_{\text {Hertz }}$ for a dent with $\delta=-1.5 \mu \mathrm{m}$. All other parameters followed Table 1 and Table 2 with PAO B. Fig. 11a shows the maximum pressures, $\hat{p}$, along each $X$ position. The figure includes the reference EHL profile for the smooth rolling cylinder at $p_{\text {Hertz }}=1.93 \mathrm{GPa}$.

Fig. 11b shows a material view at the dent of the maximum Findley index for all $y$-coordinates. The dent profile is included for the reference position. The curve sections with low and constant $F i$-values are the fatigue prediction for the respective smooth cylinder contact. The lowest $p_{\text {Hertz }}$ barely increased the fatigue risk at the dent compared with the smooth cylinder. For higher $p_{\text {Hertz }}$, the $F i$-peak developed on the left side in the dent due to the EHL interaction.

The pressure increase around the dent was smaller than for the asperity and not constant as in Fig. 7. For the $-1.5 \mu \mathrm{m}$ dent, $p_{\text {Hertz }}$ had to be at least $1.5 \mathrm{GPa}$ for the pressure increase to level off towards a $1.3 \mathrm{GPa}$ increase in Fig. 11c. Although the $F i$ was smaller for the dent in Fig. $10 \mathrm{~d}$ than for the corresponding asperity in Fig. $6 \mathrm{~b}$ it did almost reach 0.6 . 

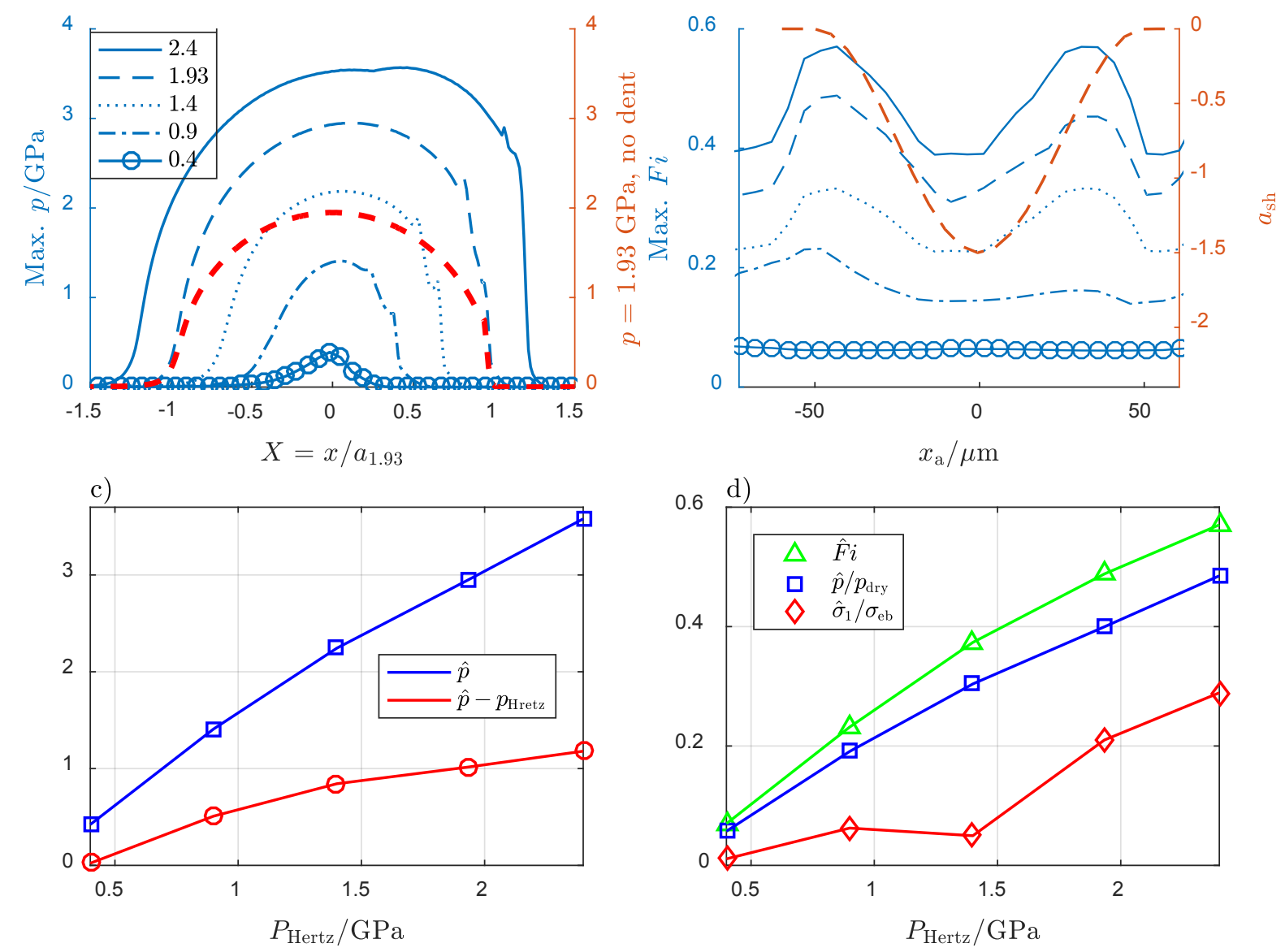

Fig. 11 The results from a dent passing through EHL contacts with different $p_{\text {Hertz. The value }}$ $p_{\text {dry }}$ refers to a dry contact with an asperity. a) $p$, b) $\hat{\sigma}_{1}$ near the asperity, c) $\hat{p}$ summary and d) fatigue risk summary.

For the investigated dent, the fatigue risk was independent of temperature, see Fig. 12. The global values $\hat{p}$ and $\hat{\sigma}_{1}$ were only marginally affected. 

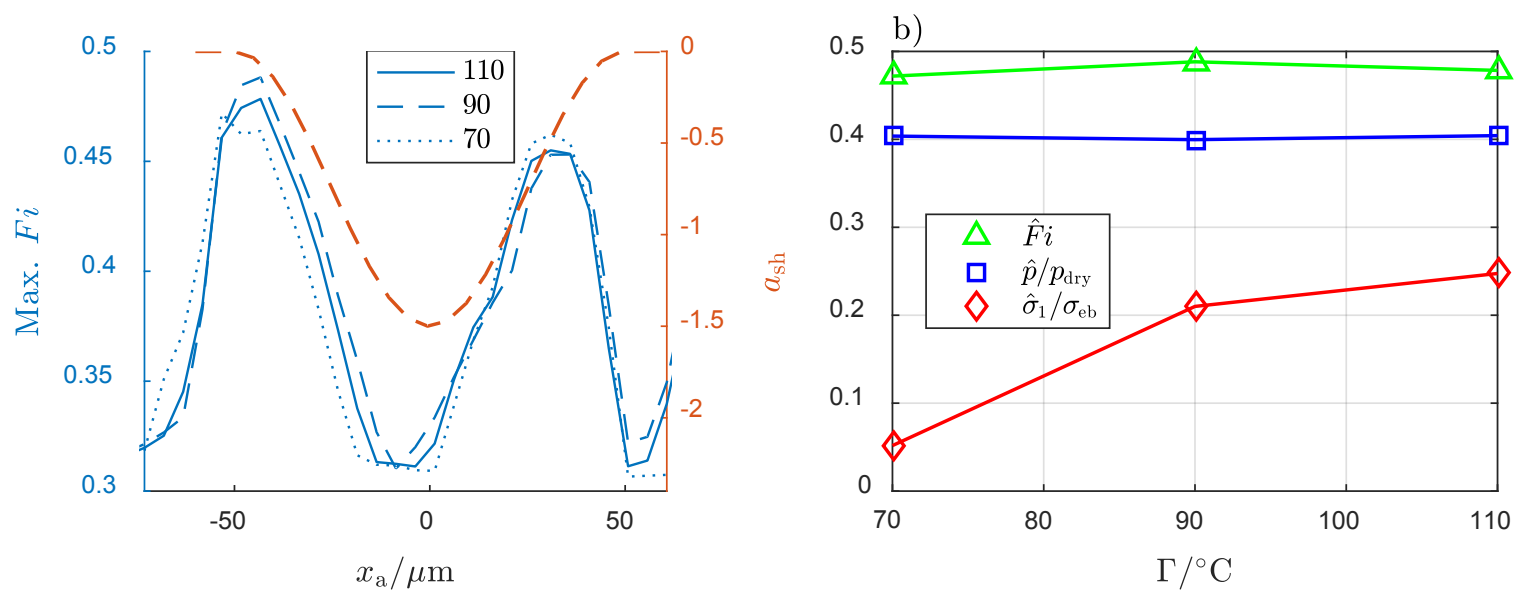

Fig. 12. Effects of contact temperature: a) Fatigue damage, maximum for all $y$. b) Maximum normalized $p, \sigma_{1}$ and $F i$.

The effects of different dent depth is illustrated in Fig. 13a and 12b. The results show that the fatigue risk increased almost linearly with the depth, corresponding to the linear increase found for the height of the asperities. However, a $1.5 \mu \mathrm{m}$ deep dent caused approximately the same fatigue damage as a $0.5 \mu \mathrm{m}$ high asperity.

In Fig. $13 \mathrm{c}$ and $\mathrm{d}$ the widths of the dents was varied. The thin dent in Fig. 13c caused higher fatigue damage at the right side of the dent centre while the wider dents caused higher damage on the left side. Similar as for the asperities, an exponential increase could be observed for decreasing dent widths, see Fig. 13d.
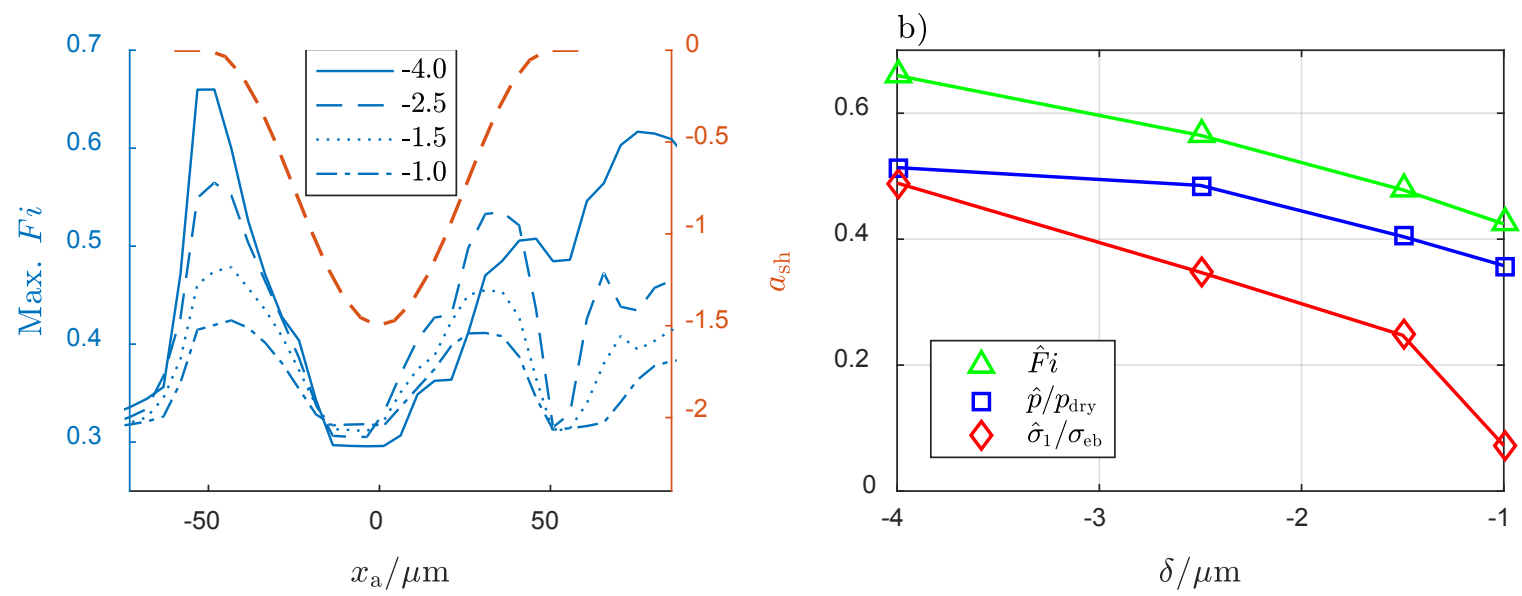

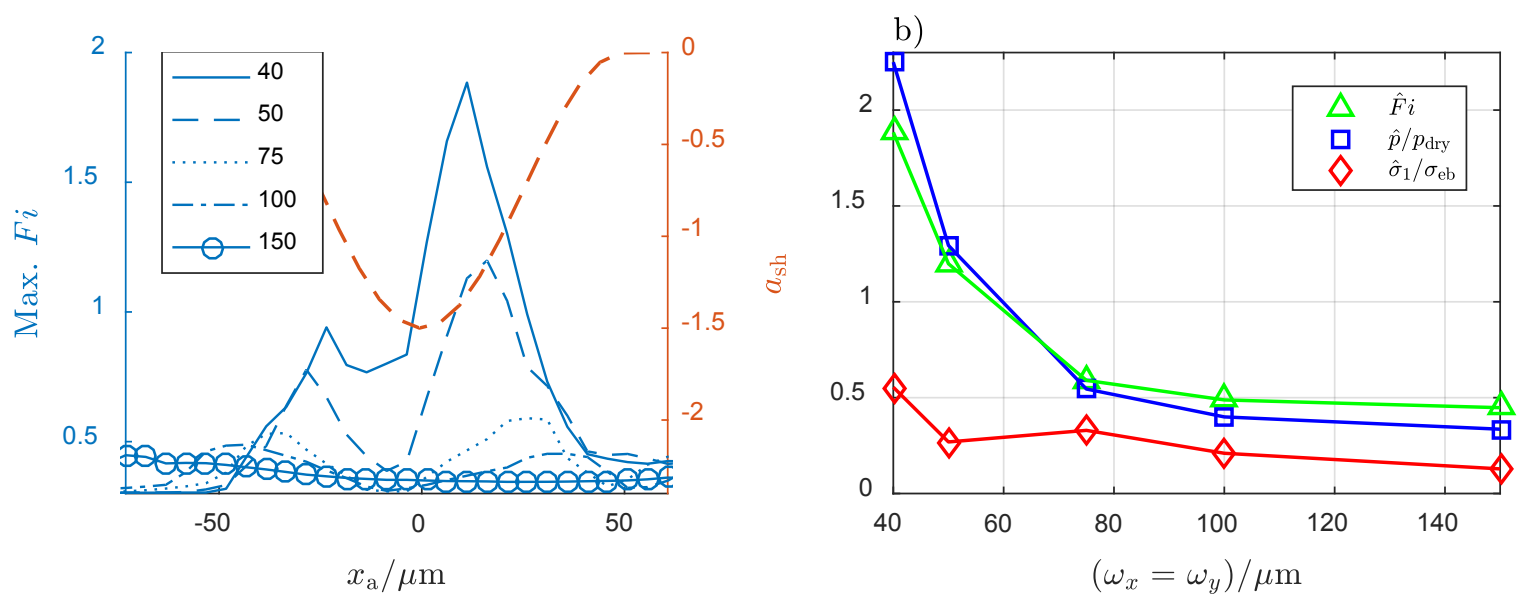

Fig. 13. The effect of dent size: a) and b) varied $\delta$ for $\omega=100 \mu \mathrm{m}$. c) and d) varied $\omega$ for $\delta=-$ $1.5 \mu \mathrm{m}$.

It can be noted that the reference dent with $\delta=-1.5 \mu \mathrm{m}$ was small compared to those that can develop from small particles in applications. Sayles and Ioannides [70] found that debris could form dents of depths up to $\delta=-5 \mu \mathrm{m}$ and widths in the range of $20 \mu \mathrm{m}<\omega<200 \mu \mathrm{m}$. The dents presented in their article had sharper edges and may therefore induce higher surface stresses, than the currently investigated sine-shape dents. Sayles and Ioannides [70] reason that a critical debris aspect ratio may exist. The decreasing slope of the damage in Fig. 13b compared with the exponential increase in Fig. 13d indicates that the severity of a dent can not be predicted by an aspect ratio alone. The amplitude has to increase far beyond $\delta=-4.0 \mu \mathrm{m}$ to initiate fatigue when $\omega=100 \mu \mathrm{m}$, i.e. the amplitude has to be far greater than 0.04 of the wavelength for this case. While at $\delta=-1.5 \mu \mathrm{m}$ a wavelength of $\omega=50 \mu \mathrm{m}$ is enough to cause damage. Here the amplitude is only 0.03 of the wavelength.

\section{Discussion}

The simulations showed that small point shaped asperities and dents can be one cause for surface initiated RCF in lubricated EHL contacts. The asperity point load mechanism provided an explanation for surface initiated RCF that is based on stresses from 3 dimensional contacts as defined by contact mechanics and the accepted knowledge on how cracks initiate and behave at ordinary fatigue. Thus, surface initiated RCF is not a separate form of fatigue; it is ordinary fatigue at special, but for rolling contacts common, load conditions. The simulations showed that slip is not necessary for RCF if sufficiently large asperities are present. Newtonian lubrication was used here since there was no global shear traction in the contact, only small shearing appeared due the Poiseuille term in Reynolds equation. The lubrication isolated the surfaces from each other except for the tip of the higher asperities where metal contact developed. Regardless of metal contact, the asperities provided a local point pressure peak which supplied local in surface tensile stresses when the asperity entered and exited the rolling nominal contact. This tensile stress component together with the large shear stress amplitude developed by the over-rolling contact provided a sufficiently large stress cycle for fatigue initiation according to the general purpose Findley multi-axial criterion for non-proportional stress cycles with material endurance data from independent uni-axial fatigue experiments.

When the results for the conditions in Table 1 and the PAO B lubricant in Table 2 were reviewed, some conditions for RCF initiation were noted. Firstly, it was noted that a point 
shaped asperity had to be present. Then, if all parameters except one was kept at the reference value, then asperity heights $\delta>1.4 \mu \mathrm{m}$ (Fig. 8b), asperity width $\omega_{x}=\omega_{y}<110 \mu \mathrm{m}$ (Fig. 8d), rolling contact pressure $p_{\text {Hertz }}>1.7 \mathrm{GPa}$ (Fig. 7b) and global temperature $\Gamma>70{ }^{\circ} \mathrm{C}$ (Fig. 6b), can initiate fatigue. Six different lubricants were reviewed, with some difference in fatigue risk, but all suggested fatigue for the reference configuration. Comparing the fatigue prediction in Fig. 5 and the lubrication data in Table 2 it appears the viscosity was the most important lubrication parameter, if one is to be singled out, for RCF. In Table 2 the lubricants are almost sorted after decreasing reference viscosity and in Fig. $5^{\mathrm{f}}$ the same sequence shows increasing fatigue risk. Thus, decreasing viscosity suggested increasing RCF risk according to the presently investigated point load hypothesis.

The results for the equally sized dents as the asperities suggested dents being less dangerous than asperities. At equal conditions, for instance $p=2 \mathrm{GPa}$ in Fig. 11d, Fi for the dent was half the value for the asperity in Fig. 7b. However, it was noted in Fig. 10b that lubrication interaction with dents clearly increased the fatigue risk compared to the smooth surface.

\subsection{Dry contact comparison}

Earlier studies have shown that small point shaped asperities can cause fatigue cracks at dry conditions [11]. Such cracks will then propagate to the archetypical surface initiated pits in Fig. 1 [7] [8] [9] [10] driven by the asperity point loads in combination with the rolling cylinder load.

The effect of the lubrication on the asperity contact is illustrated in Fig. 14a-d by comparing dry and EHL contact pressures for three different asperity positions. The EHL simulations were run with the lubrication parameters of PAO B in Table 2 and the loading conditions in [7].

The solid lines in Fig. 14a represent $X_{d}=-1.2$, the dashed lines $x_{d}=-0.94$ and the dotted lines were obtained when $X_{d}=0$. In the EHL contact the asperity pressure does not reach quite the same height as in the dry contact case, the squeeze effect is visible in front of the asperity and the pressure decrease just beside the asperity is smaller. In the dry contact, a larger pressure peak is visible at first interaction between asperity and cylinder. Fig. 14b and c illustrates the pressure at the asperity when it has entered the EHL contact, $X_{d}=-0.91$, and at the exit $X_{d}=0.91$. The cylinder contact rim at $X= \pm 1$ is indicated with a dashed white line at respective the figure edge. At entry, the squeeze effect is visible as the pressure increase 60 $\mu \mathrm{m}$ to the right of the asperity. At the exit region the EHL pressure distribution was very similar to the dry case [7], but with almost $1 \mathrm{GPa}$ lower asperity pressure. For the dry contact the pressure results would be symmetric in entry and exit since there would be no squeeze effect or EHL pressure spike. When the dry and the EHL contacts with point asperities were compared, the important conclusion was that all major features remained for the high pressure spike. Thus, the propagation results for a crack at the asperity in dry conditions were expected to remain for EHL contacts.

The maximum EHL pressure on the dent of $\delta=-1.0 \mu \mathrm{m}$ during a load cycle is presented in Fig. 14d. The pressure distribution is much less symmetric with respect to the transverse centre line than for the asperity in Fig. 14a. Along the left dent rim $p$ increased compared to the surrounding $p$ whereas it decreased along the right rim. For dry conditions a small symmetric pressure increase has been reported symmetrically around the dent rim [11]. Thus, the interaction between the dent and the EHL contact may produce a noticeable pressure spike which is absent for the dry contact. 

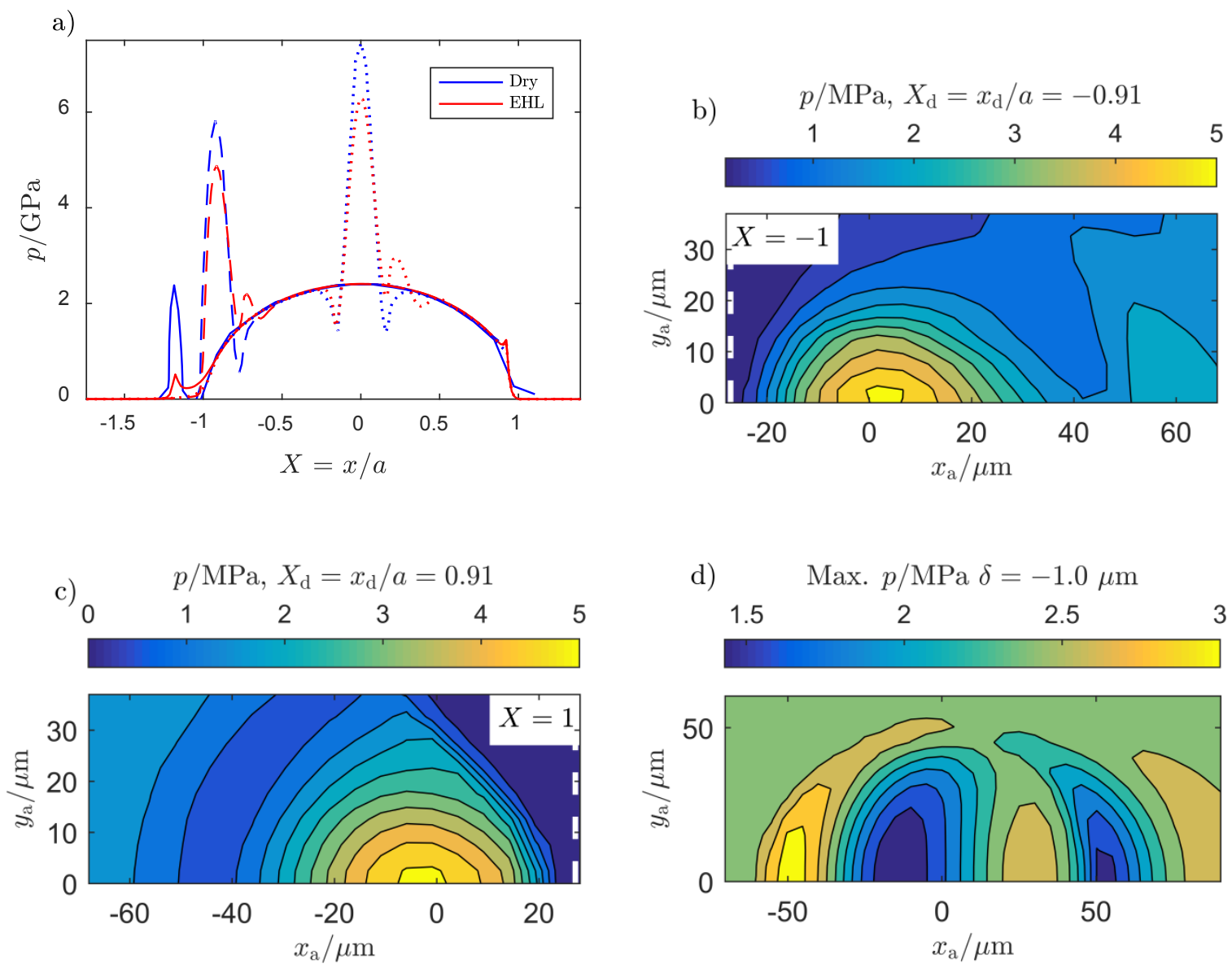

Fig. 14. a) Dry and EHL contact $p$ with asperity at 3 positions. b) EHL $p$ at asperity entry. c) EHL $p$ at asperity exit. d) Maximum EHL $p$ around a dent during cycle. Data from Table 1.

\subsection{The Fatigue evaluation}

Fatigue was investigated in the material view where $F i$ summarized the fatigue risk in each material point from the whole load cycle. The contact view, such as Figs $4 \mathrm{a}$ and $4 \mathrm{~b}$ supported the Findley result by illustrating the stress distributions at the instant where and when during the load cycle a high stress occurred. The fatigue criterion in Eq. (15) contains two parts, the shear stress amplitude on the critical plane $\tau_{\text {amp }}$ and the maximum normal stress sometime during the load cycle on that plane $\sigma_{\mathrm{n} \text {,max }}$. For some deeper understanding, the Findley graph in Fig. 4d was split into its two components in Figs 14a and 14b including arrows showing the normal to the plane with the $F i$ value. The arrows are presented for $\sigma_{\mathrm{n}, \max }>200 \mathrm{MPa}$ and $\tau_{\mathrm{amp}}$ $>250 \mathrm{MPa}$. Thus, $F i$ was determined by both $\sigma_{\mathrm{n}, \max }$ and $\tau_{\mathrm{amp}}$. The highest $F i$ was, as in Fig. 4d, observed on the transverse sides of the asperities which agreed with the large $\sigma_{\mathrm{n}, \max }$ in Fig. 15a. The corresponding largest $\tau_{\mathrm{amp}}$ in Fig. 15b was however found on the symmetry line with $Y=\mathrm{o}$ and to the left of $X=\mathrm{o}$. For the asperity, $\sigma_{\mathrm{n}, \max }$ had a large influence due to the large radial tensile surface stress from the asperity point load, Fig. $3 \mathrm{c}$, and the large coefficient $\kappa$ in Eq. (15).

For the dents, the position with the highest $F i$ was always on the symmetry line and most often at the right side of the dent, see Figs 10d, 11b, 12a, 13a and 13c. The Fi result in Fig. 10d was divided into its components in Fig. 16. The figure shows that for the dent, the major part 
of the fatigue value was due to $\tau_{\mathrm{amp}}$. $\sigma_{\mathrm{n}, \max }$ only gave a minor contribution to the fatigue damage at the dent. However, it is tensile at $\widehat{F}_{l}$ in Fig. 10d, which is a requirement for opening a fatigue crack.

Considering Figs 15 and 16 it was deduced that contact fatigue at asperities and dents have different explanations. At asperities, fatigue was primarily caused by the in surface tensile stress from the asperity point load. At dents, the lubrication interaction with the walls created the pressure and stress peak that gave large $\tau_{\text {amp }}$ for the highest $F i$.
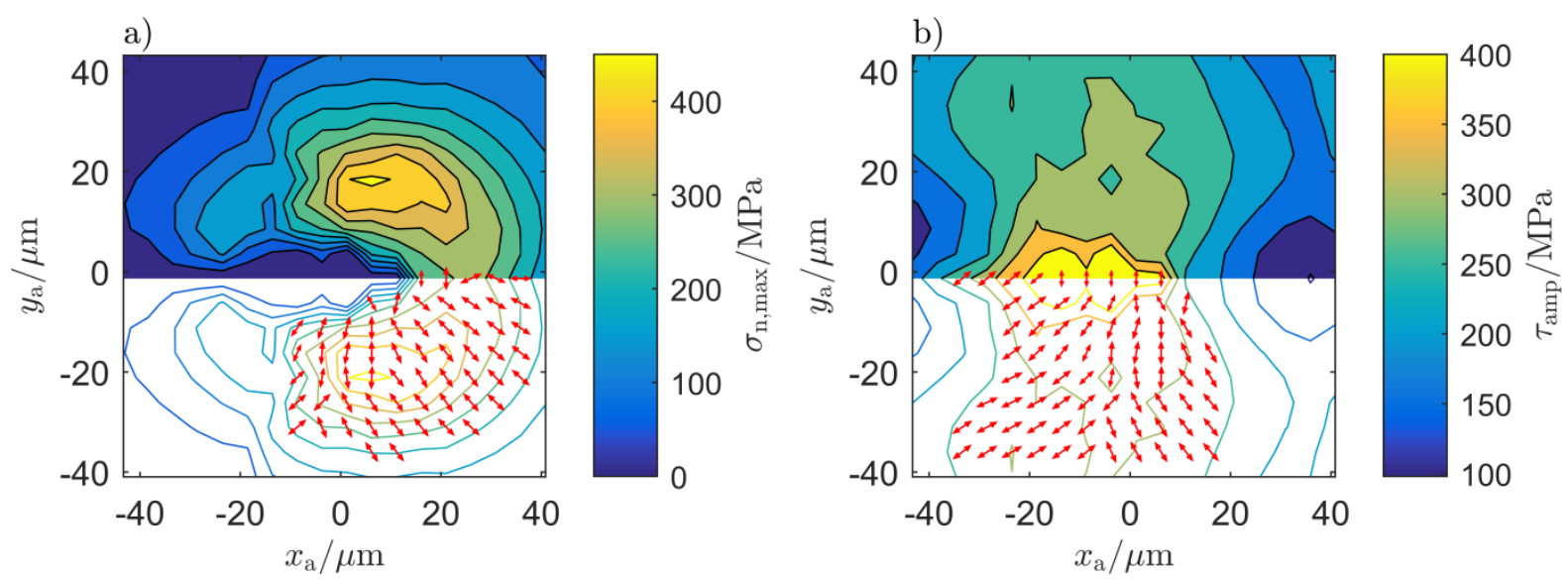

Fig. 15. Findley components in Eq. (15) for the asperity in Fig. 4d. a) The maximum normal stress, $\left.\sigma_{\mathrm{n}, \max } . \mathrm{b}\right)$ The shear stress amplitude, $\tau_{\mathrm{amp}}$.
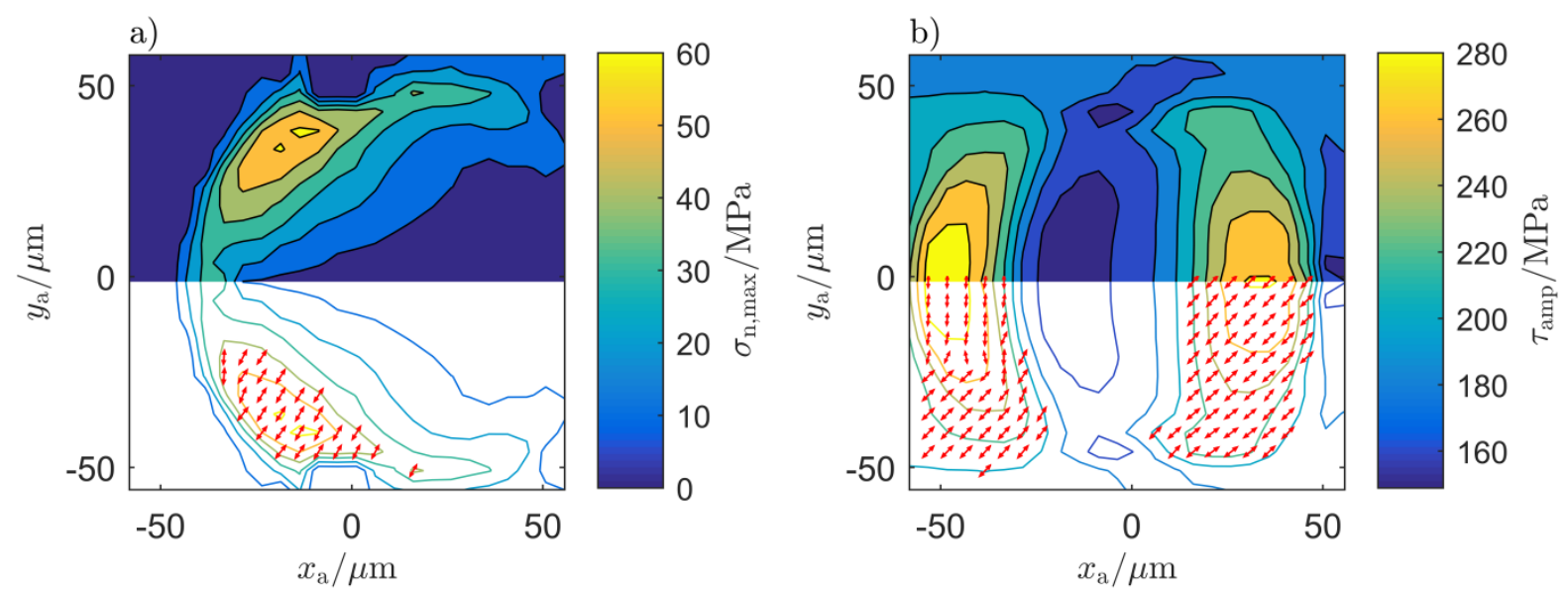

Fig. 16. Findley components for the dent in Fig. 10d. a) The maximum normal stress, $\sigma_{\mathrm{n}, \max }$ b) The shear stress amplitude, $\tau_{\mathrm{amp}}$.

\subsection{The $\Lambda$-ratio}

The risk for RCF in EHL contacts is often estimated with the $\Lambda$-ratio, $\Lambda=h_{\min } / R_{\mathrm{a}}$. For the present surface with a single asperity or dent, $R_{\mathrm{a}}$ was ambiguous. The peak to valley distance $R_{\mathrm{t}}=|\delta|$ was well defined but clearly too large for comparisons with $R_{\mathrm{a}}$ based results in the 
literature. A simple relation $R_{\mathrm{a}}=R_{\mathrm{t}} / 4$ was therefore determined by computing the expected $R_{\mathrm{a}}$ for a constant roughness ansatz over $R_{\mathrm{t}}$. Thus,

$$
\Lambda=\frac{4 h_{\min }}{|\delta|}
$$

was used here. A $\Lambda$-ratio below 1 typically indicates increased risk for asperity contact and RCF. Conversely, a value above 1 indicates a fairly low risk for RCF, see for example [71]. The $\Lambda$-ratio for all presently simulated contacts is compared to the $F i$ value in Fig. 17a. Open markers are results for asperities and solid markers are for dents. A linear relation with negative slope between $\Lambda$ and $F i$ in Fig. 17a suggested correlation between $\Lambda$ and $F i$ with respect to the investigated parameter, see for instance $p_{\text {Hertz }}, \delta, \Gamma$ and lubricant, i.e. $\eta_{\mathrm{o}}$. The series with varying asperity width $\omega$ and $\omega_{x} / \omega_{y}$ had constant $\Lambda$ but clearly varying fatigue risk $\mathrm{Fi}$. Fig. 17b shows a comparison between $\Lambda$ and maximum $\sigma_{1} / \sigma_{\mathrm{eb}}$ during the load cycle with the same conclusions regarding parameter correlation. The dents gave the same $\Lambda$-ratio but almost half the $F i$ and a third of $\sigma_{1} / \sigma_{\mathrm{eb}}$ for the asperity.
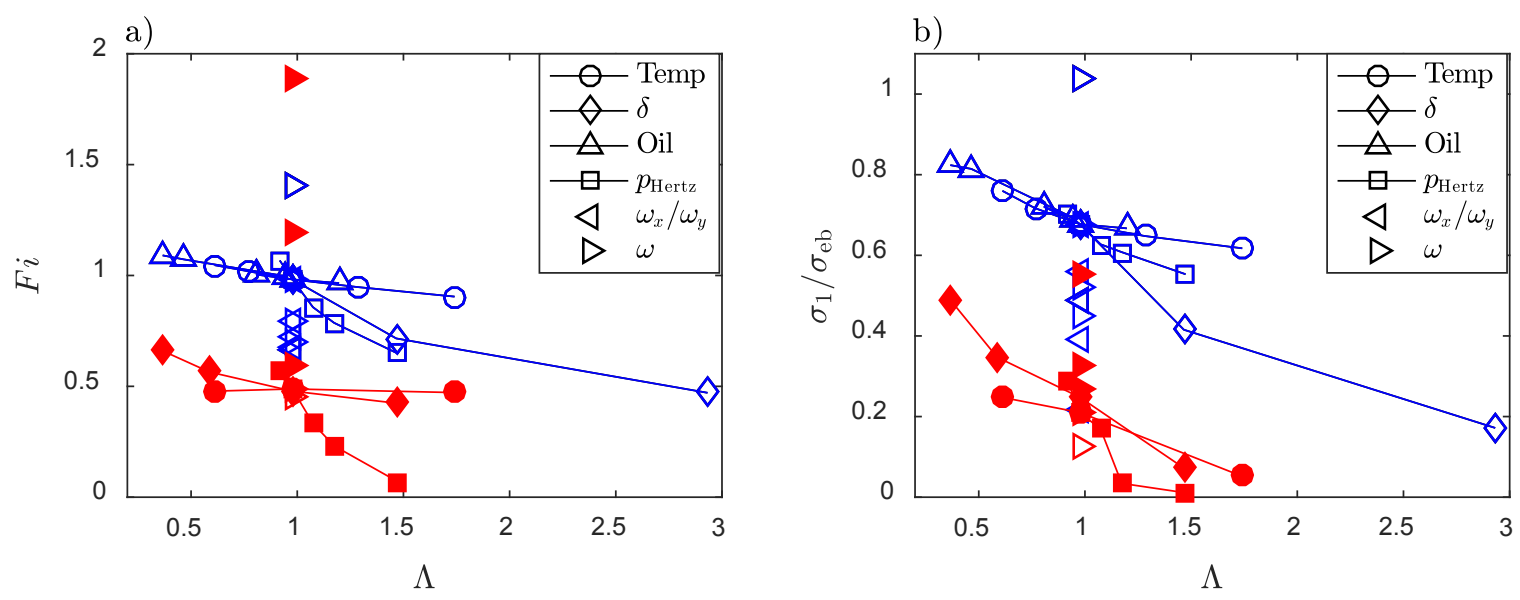

Fig. 17. Correlation between $\Lambda$ and a) $F i$ and b) $\sigma_{1} / \sigma_{\mathrm{eb}}$. Open blue and solid red symbols represent asperities and dents, respectively.

The varying RCF risk for equal $\Lambda$ in Fig. 17 and in particular the difference in risk between equally large and equally shaped asperities and dents illustrated that the $\Lambda$-ratio or $R_{\mathrm{a}}$ alone may be misleading in predicting RCF risk. The detrimental difference between asperities and dents for equal $R_{\mathrm{a}}$ can be identified by also considering the surface skewness or third statistical moment of the roughness

$$
R_{\mathrm{sk}}=\frac{1}{L R_{q}^{3}} \int_{0}^{L} z^{3}(x) d x
$$

where $L$ is the measurement length and $R_{\mathrm{q}}$ is the root mean square roughness. Positive $R_{\mathrm{sk}}$ indicates asperities and negative $R_{\mathrm{sk}}$ suggests dents. Hence a positive $R_{\mathrm{sk}}$ increases the RCF risk for equal $R_{\mathrm{a}}$. 


\section{Conclusion}

Point type asperities and dents gave pressure spikes and substantial tensile in surface stresses on the sides of the asperities and inside the dents when they were over-rolled by an otherwise smooth and lubricated cylinder. The detailed investigation of single asperities and dents led to the conclusion that stress peaks at asperities and dents were the results of different mechanisms.

The point type pressure spike at asperity entry and exit was particularly important since it gave rise to stress cycles with large tensile stress maxima in combination with large shear stress amplitudes in the surface and following gave decisive contribution to the fatigue risk at the irregularities as they entered and exited the rolling contact. The simulations resulted in a series of conclusions for RCF initiation at point shaped asperities.

- At asperity entry and exit of the EHL contact the pressure spike remained almost as severe as for the dry contact, at $87 \%$ of the dry value.

- Asperities in ground gear surfaces can be sufficiently large to initiate contact fatigue in lubricated rolling contacts without slip.

- The predicted fatigue risk became substantial for axial-symmetric asperities with height $\delta=1.5 \mu \mathrm{m}$, width $\omega=100 \mu \mathrm{m}$ and $p_{\text {Hertz }}>2 \mathrm{GPa}$.

- The maximum risk for fatigue and crack initiation was found on the transverse sides of the asperities. Analysis of the Findley fatigue criterion suggested that the tensile insurface stress played a vital role in the fatigue risk at asperities.

- The maximum pressure on the asperity increased with around 3.5-4 GPa for all simulated $p_{\text {Hertz. }}$

- The $\Lambda=4 h_{\min } / \delta$ indicated the severity of the contact for some parameters, such as $p_{\text {Hertz }}, \delta, \Gamma$ and $\eta_{\mathrm{o}}$. For other parameters the $\Lambda$-ratio can be misleading.

For dents another mechanism was the cause for the point type pressure spike. The EHL interacted with the dents to create pressure spikes on the inside of the dent rim and possible fatigue initiation on the inner sides of the dents. The highest fatigue risk at the dent was found due to the point pressure spike.

- Dents yielded about half the fatigue risk expressed in $F i$ as the correspondingly high asperities.

- The maximum fatigue risk of a dent developed on the inside slope and along the rolling symmetry line of the dent.

The main research goal was met: EHL simulations with load, asperity size and material fatigue data from the application in Fig. 1 predicted RCF using general purpose multi-axial fatigue criterion. Thereby the asperity point load mechanism was successfully verified for surface initiated RCF close to the gear pitch line in the application. 


\section{Acknowledgement}

The authors gratefully acknowledge financial support from The Swedish Research Council [grant number 2012-5922]. The Swedish Research Council had no involvement in the preparation of the article. The authors thank Mr. E. Nordin and Dr. M. Henriksson for providing the gear geometry data in Table 1 and data for top part of Fig. 2a.

\section{References}

[1] S. Way, "Pitting due to Rolling Contact," Journal of applied mechanics, pp. 49-58, 1935.

[2] T. Tallian, Failure Atlas for Hertz Contact Machine Elements, New York: ASME Press, 1992.

[3] M. MackAldener, M. Olsson, "Tooth interior fatigue fracture - computational and material aspects.," International Journal of Fatigue, vol. 23, pp. 329-340, 2001.

[4] “Ansol.us," Advanced Numerical Solutions LLC, [Online]. Available: http://ansol.us/Products/Helical3D/.

[5] M. Olsson, "Contact fatigue and tensile streses," Engineering Against Fatigue, pp. 651-657, 1999.

[6] J. Dahlberg, B. Alfredsson, "Influence of a single axisymmetric asperity on surface stresses during dry rolling contact," International Journal of Fatigue, vol. 29, pp. 909-921, 2007.

[7] D. Hannes, B. Alfredsson, "Rolling contact fatigue crack path prediction by the asperity point load mechanism," Engineering Fracture Mechanics, vol. 78, no. 17, pp. 2848-2869, 2011.

[8] D. Hannes, B. Alfredsson, "A fracture mechanical life prediction method for rolling contact fatigue based ont he asperity point load mechanism," Engineering Fracture Mechanics, vol. 83, pp. 6274, 2012.

[9] D. Hannes, B. Alfredsson, "Surface initiated rolling contact fatigue based on the asperity point load mechanism - A parameter study," Wear, vol. 294, pp. 457-468, 2012.

[10] D. Hannes, B. Alfredsson, "Nummerical investigation of the spall opening angle of surface initiated rolling contact fatigue," Engineering Fracture Mechanics, vol. 131, pp. 538-556, 2014.

[11] J. Dahlberg, B. Alfredsson, "Surface stresses at an axisymmetric asperity in a rolling contact with traction," Int. Journal of Fatigue, vol. 30, pp. 1606-1622, 2008.

[12] B. Alfredsson, M. Olsson, "Inclined Standing Contact Fatigue and its Relation to Surface Distress," in Fatigue 2002: Proceedings of the Eight International Fatigue Congress, Stockholm, 2002.

[13] B. Alfredsson, M. Olsson, "Inclined standing contact fatigue," Fatigue and Fracture of Engineering Materials and Structures, vol. 28, pp. 589-602, 2003.

[14] A. Mohrenstein-Ertel, Die Berechnung der hydrodynamischen Schmierung gekrümmter Oberflächen unter hoher Belastung und Relativbewegung, Düsseldorf: VDI-Verlag, 1984.

[15] E. Popova, V.L. Valentin, "On the history of elastohydrodynamics: The dramatic destiny of Alexander Mohrenstein-Ertel and his contribution to the theory and practice of lubrication.," ZAMM-Journal of Applied Mathematics and Mechanics/Zeitschrift für Angewandte Mathematik und Mechanik, vol. 95, no. 7, pp. 652-663, 2015.

[16] A. Petrusevich, "Fundamental Conclusions from the Contact Hydrodynamic Theory of Lubrication," Izvestiya Akademii Nauk SSR, pp. 209-223, 1951.

[17] D. Dowson, G.R. Higginsson, "A numerical soluion to the elasto-hydrodynamic problem," Mechanical Engineering Science, vol. 1, pp. 6-15, 1959.

[18] D. Dowson, G.R. Higginson, A.V. Whitaker, "Elasto-hydrodynamic lubrication: A survey of isothermal solutions," Journal of Mechanical Engineering Science, vol. 4, no. 2, pp. 121-126, 1962.

[19] B.J. Hamrock, D. Dowson, "Isothermal elastohydrodynamic lubrication of point contacts," Lubrication Technology, pp. 223-228, 1976.

[20] D. Dowson, P. Ehret, "Past, present and future studies in elastohydrodynamics".

[21] H. Spikes, "Sixty years of EHL," Lubrication Science, vol. 18, no. 4, pp. 265-291, 2006. 
[22] P. M. Lugt, G.E. Morales-Espejel, “A review of elasto-hydrodynamic lubrication theory,” Tribology Transactions, vol. 54, no. 3, pp. 470-496, 2011.

[23] C.H. Venner, A.A. Lubrecht, Multilevel Methods in Lubrication, Elsevier, 2000.

[24] M. Masjedi, M.M. Khonsari, "On the Effect of Surface Roughness in Point-Contact EHL: Formulas for Film Thickness and Asperity Load," Tribology International, vol. 82, pp. 228-244, 2015.

[25] A. Rosenkranz, A. Szurdak, C. Gachot, G. Hirt, F. Mücklich, "Friction reduction under mixed and full film EHL induced by hot micro-coined surface patterns," Tribology International, vol. 95, pp. 290-297, 2016.

[26] P. Šperka, I. Křupka and M. Hartl, "Experimental Study of Roughness Effect in a Rolling-Sliding EHL Contact. Part II: Complementary Effects,” Tribology Transactions, vol. 59, no. 2, pp. 277285, 2016.

[27] I. Křupka, P. Šperka and M. Hartl, "Effect of surface roughness on lubricant film breakdown and transition from EHL to mixed lubrication," Tribology International, vol. 100, pp. 116-125, 2016.

[28] H. Elrod, "A review of theories for the fluid dynamic effects of roughness on laminar lubricating films," Columbia University, New York, 1977.

[29] H.S. Cheng, M. Bali, "Stress distributions around furrows and asperities in EHL line contacts," The American society of mechanical engineers, 1980.

[30] A.A. Lubrecht, R.S. Dwyer-Joyce, E. Ioannides, "Analysis of the influence of indentations on contact life," Proc. of 18th Leeds-Lyon Symposium on tribology, 1991.

[31] B.R. Höhn, K. Michaelis, O. Keril, "Influence of Surface roughness on pressure distribution and film thickness in EHL-contacts," Tribology International, vol. 39, pp. 1719-1725, 2006.

[32] D. Epstein, L. M. Keer, Q. J. Wang and H. S. Cheng, "Effect of Surface Topography on Contact Fatigue in Mixed Lubrication," Tribology Transactions, vol. 46, pp. 506-513, 2003.

[33] N. Ren, D. Zhu, W. W. Chen, Y. Liu, Q. J. Wang, "A Three-dimensional Deterministic Model for Rough Surface Line-Contact EHL Problems,” Journal of Tribology, vol. 131, 2009.

[34] S. Li, A. Kahraman, "A fatigue model for contacts under mixed elastohydrodynamic lubrication condition," International Journal of Fatigue, vol. 33, pp. 427-436, 2011.

[35] E. Ioannides, T.A. Harris, “A New Fatigue Life Model for Rolling Bearings," Journal of Tribology, vol. 107, no. 3, pp. 367-377, 1985 .

[36] E. Zaretsky, "Fatigue Criterion to System Design, Life, and Reliability," Journal of Propulsion and Power, vol. 3, no. 1, pp. 76-83, 1987.

[37] D. Zhu, N. Ren, Q.J. Wang, "Pitting Life Prediction Based on a 3D Line Contact Mixed EHL Analysis and Subsurface von Mises Stress Calculation," Journal of Tribology, vol. 131, no. 4, 2009.

[38] H. Qiao. H.P. Evans, R.W. Snidle, "Comparison of fatigue model results for rough surface elastohydrodynamic lubrication," J. Engineering Tribology, vol. Vol. 222 Part J, no. Proc. IMechE, pp. 381-393, 2008.

[39] G. Xu, F. Sadeghi, J.D. Cogdell, "Debris Denting Effects on Elastohydrodynamic Lubricated Contacts," Journal of Tribology, vol. 119, pp. 579-587, 1997.

[40] M. Jiang, L.Gao, P. Yang, Z.M. Jin, D. Dowson, "Numerical analysis of the thermal micro-EHL problem of point contact with a single surface bump," Life Cycle Tribology, pp. 627-635, 2005.

[41] N. Deolalikar, F. Sadeghi, S. Marble, "Nummerical Modeling of Mixed Lubrication and Flash Temperature in EHL Elliptical Contacts," Journal of Tribology, vol. 130, no. 1, 2008.

[42] C.J. Hooke, K.Y. Li, G. Morales-Espejel, "Rapid calculation of the pressures and clearances in rough, rolling-sliding elastohydrodynamically lubricated contacts. Part 2: general, non-sinusoidal roughness," J. Mechanical Engineering Science, vol. Vol. 221 Part C, pp. 551-564, 2007.

[43] Y. Liu, S. Mahadevan, "A unified multiaxial fatigue damage model for isotropic and anisotropic materials," Int. Journal of Fatigue, vol. 29, no. 2, pp. 347-359, 2007.

[44] D. Nelias, F. Ville, "Detrimental Effects of Debris Dents on Rolling Contact Fatigue," Journal of Tribology, vol. Vol. 122, pp. 55-64, 200.

[45] G.E. Morales-Espejel, A. Gabelli, "The Progress of Surface Rolling Contact Fatigue Damage of 
Rolling Bearings with Artificial Dents,” Tribology Transactions, vol. 58, pp. 418-431, 2014.

[46] G. Morales-Espejel, "Surface roughness effects in elastohydrodynamic lubrication: A review with contributions," Journal of Engineering Tribology, vol. 228, no. 11, pp. 1217-1242, 2014.

[47] T. Ahlroos, H. Ronkainen, A. Helle, R. Parikka, J. Virta and S. Varjus, "Twin disc micropitting tests," Tribology International, vol. 42, pp. 1460-1466, 2009.

[48] K.J. Sharif, H.P. Evans, R.W. Snidle, "Modelling of elastohydrodynamic lubrication and fatigue," Engineering Tribology, pp. 1039-1050, 2012.

[49] W. Findley, A theory for the effect of mean stress on fatigue of metals under combined torsion and axial load of bending, Providence: Eng. Mat. Research Lab., Div. of Eng., 1958.

[50] B. Alfredsson, M. Olsson, "Applying multiaxial fatigue criteria to standing contact fatigue," International Journal of Fatigue, vol. 23, no. 6, pp. 533-548, 2001.

[51] B. Alfredsson, A. Cadario, "A study on fretting friction evolution and fretting fatigue crack," International Journal of Fatigue, vol. 26, pp. 1037-1052, 2004.

[52] O. Reynolds, "On the Theory of Lubrication and Its Application to Mr. Beauchamp Tower's Experiments, Including an Experimental Determination of the Viscosity of Olive Oil," Philosophical Transactions of the Royal Society of London, vol. 177, pp. 157-235, 1886.

[53] M. Sosa, "Running-in of gears from a surface transportation and efficiency point of view," KTH, Stockholm, 2015.

[54] H.P. Evans, R.W. Snidle, K.J. Sharif, M.J. Bryant, "Predictive modelling of fatigue failure in concentrated lubricated contacts," Faraday discussions , vol. 156, no. 1, pp. 105-121, 2012.

[55] A. Clark, I.J.J. Weeks, R.W. Snidle, H.P. Evans, "Running-in and micropitting behaviour of steel surfaces under mixed lubrication conditions," Tribology International, vol. 101, pp. 59-68, 2016.

[56] K. Johnsson, Contact mechanics, Cambridge University Press, 2003.

[57] R. Larsson, P.O. Eriksson, E. Sjöberg and E Höglund, "Lubricant properties for input to hydrodynamic and elastohydrodynamic lubrication analyses," Journal of Engineering Tribology, vol. 214, no. 1, pp. 17-27, 2000.

[58] C. Roelands, "Correlational Aspects of the Viscosity-Temperature Pressure Relationship of Lubricating Oils," Delft University, 1966.

[59] H. Salehizadeh, N. Saka, "Thermal non-Newtonian elastohydrodynamic lubrication of rolling line contacts," Journal of tribology, vol. 113, no. 3, pp. 481-491, 1991.

[6o] R. Gohar, Elastohydrodynamics, Chichester: Ellis Horwood Limited, 1988.

[61] S. Li, A. Kahraman, "A physics-based model to predict micro-pitting lives of lubricated point contacts," International Journal of Fatigue, vol. 47, pp. 205-215, 20013.

[62] D.F. Socie, G.B. Marquis, "Multiaxial Fatigue," Warrendale, Pa, Society of Automotive Engineers, 2000, pp. 138-141.

[63] M. Shirzadegan, A. Almqvist, R. Larsson, "Fully deterministic nummerical simulation of cam and roller follower.," Luleå University of Technology, Luleå, 2015.

[64] P. Huang, Nummerical calculations of lubrication: methods and programs, Guangzhou, China: John Wiley \& Sons, 2013.

[65] P. Huang, Numerical Calculation of Elastohydrodynamic Lubrication: Methods and Programs, Wiley, 2015.

[66] D. Zhu, T.Z. Hu, "The Study of Transition from Elastohydrodynamic to Mixed and Boundary Lubrication," Proceedings of the 1999 STLE/ASME HS Cheng Tribology Surveillance, pp. 150$156,1999$.

[67] A. Love, "The stress produced in a semi-infinite solid by pressure on part of the boundary," Phil. Trans. R. Soc. Lond., vol. 228, pp. 377-420, 1929.

[68] M.J.A. Holmes, H.P. Evans, T.G. Hughes, R.W. Snidle, "Transient elastohydrodynamic point contact analysis using a new coupled differential deflection method, Part1: theory and validation," Journal of Engineering Tribology, vol. 217, no. 4, pp. 289-303, 2003.

[69] B. Alfredsson, M. Olsson, "Standing contact fatigue," Fatigue and Fracture of Eng. Mat. and 
Struct. , vol. 22, no. 3, pp. 225-237, 1999.

[70] R.S. Sayles, E. Ioannides, "Debris damage in rolling bearing and its effect on fatigue life," ASME, Journal of Tribology, vol. 110, no. 1, pp. 26-31, 1988.

[71] A. Clarke, H.P. Evans, R.W. Snidle, "Understanding micropitting in gears," Journal of mechanical engineering science, vol. 230, no. 7-8, pp. 1276-1289, 2016.

[72] S. Preston, "Bending fatigue strength of carburising steel SS2506," Material Science and Technology, vol. 7, pp. 105-110, 1991.

[73] C.H. Venner, A.A. Lubrecht, "Nummerical Simulation of a Transverse Ridge in a Circular EHL Contact Under Rolling/Sliding,” Journal of Tribology, vol. 116, pp. 751-761, 1994. 


\section{Appendix A. stress calculations}

The stresses inside the substrate from the EHL pressure and shear traction were obtained from a summation of stresses due to line and point pressures; line and point shear tractions. The Flamant solution for $p_{\min } \Delta x$ at $x=0$ gives

$$
\begin{aligned}
& \sigma_{x}(x, z)=-\frac{2 p_{\min } \Delta x}{\pi} \frac{x^{2} z}{\left(x^{2}+z^{2}\right)^{2}} \\
& \sigma_{y}(x, z)=v\left[\sigma_{x}(x, z)+\sigma_{z}(x, z)\right] \\
& \sigma_{z}(x, z)=-\frac{2 p_{\min } \Delta x}{\pi} \frac{z^{3}}{\left(x^{2}+z^{2}\right)^{2}} \\
& \tau_{x z}(x, z)=-\frac{2 p_{\min } \Delta x}{\pi} \frac{x z^{2}}{\left(x^{2}+z^{2}\right)^{2}} \\
& \tau_{x y}(x, z)=\tau_{y z}(x, z)=0
\end{aligned}
$$

and the solution for traction $\tau_{\mathrm{xzmin}} \Delta x$ at $x=z=0$ gives

$$
\begin{aligned}
& \sigma_{x}(x, z)=\frac{2 \tau_{\mathrm{xzmin}} \Delta x}{\pi} \frac{x^{3}}{\left(x^{2}+z^{2}\right)^{2}} \\
& \sigma_{y}(x, z)=\frac{v}{1-2 v}\left(\sigma_{x}(x, z)+\sigma_{z}(x, z)\right) \\
& \sigma_{z}(x, z)=\frac{2 \tau_{\mathrm{xz} \min } \Delta x}{\pi} \frac{x z^{2}}{\left(x^{2}+z^{2}\right)^{2}} \\
& \tau_{x z}(x, z)=\frac{2 \tau_{\mathrm{xz} \min } \Delta x}{\pi} \frac{x^{2} z}{\left(x^{2}+z^{2}\right)^{2}} \\
& \tau_{x y}(x, z)=\tau_{y z}(x, z)=0
\end{aligned}
$$

At $x=z=0$ the Flamat and line traction solutions tend to infinity, which was handled by summation of point loads. The Boussinesq solution for a point load $p \Delta x \Delta y$ at $(0,0,0)$ gives 


$$
\begin{aligned}
& \sigma_{x}(x, y, z)=\frac{p \Delta x \Delta y}{2 \pi}\left[\frac{1-2 v}{r_{\mathrm{s}_{1}}{ }^{2}}\left\{\left(1-\frac{z}{r_{\mathrm{s}_{2}}}\right) \frac{x^{2}-y^{2}}{r_{\mathrm{s}_{1}}{ }^{2}}+\frac{z y^{2}}{r_{\mathrm{s}_{2}}{ }^{2}}\right\}-\frac{3 z x^{2}}{\left.r_{\mathrm{s}_{2}}{ }^{5}\right]}\right. \\
& \sigma_{y}(x, y, z)=\frac{p \Delta x \Delta y}{2 \pi}\left[\frac{1-2 v}{r_{\mathrm{s}_{1}}{ }^{2}}\left\{\left(1-\frac{z}{r_{\mathrm{s}_{2}}}\right) \frac{y^{2}-x^{2}}{r_{\mathrm{s}_{1}}{ }^{2}}+\frac{z x^{2}}{r_{\mathrm{s}_{2}}{ }^{2}}\right\}-\frac{3 z y^{2}}{r_{\mathrm{s}_{2}}{ }^{5}}\right] \\
& \sigma_{z}(x, y, z)=\frac{3 p \Delta x \Delta y}{2 \pi} \frac{z^{3}}{r_{s_{2}}{ }^{5}}\left[\frac{p \Delta x \Delta y}{2 \pi}\left[\frac{1-2 v}{r_{\mathrm{s}_{1}}{ }^{2}}\left\{\left(1-\frac{z}{r_{\mathrm{s}_{2}}}\right) \frac{2 x y}{r_{\mathrm{s}_{1}}{ }^{2}}+\frac{x y z}{r_{\mathrm{s}_{2}}{ }^{3}}\right\}-\frac{3 x y z}{r_{\mathrm{s}_{2}}{ }^{5}}\right]\right. \\
& \tau_{x z}(x, y, z)=-\frac{3 p \Delta x \Delta y}{2 \pi} \frac{x z^{2}}{r_{\mathrm{s}_{2}}{ }^{5}} \\
& \tau_{y z}(x, y, z)=-\frac{3 p \Delta x \Delta y}{2 \pi} \frac{y z^{2}}{r_{\mathrm{s}_{2}}{ }^{5}}
\end{aligned}
$$

The singularity in Eq. A3 at $(0,0,0)$ was handled by setting

$$
\begin{aligned}
& \sigma_{x}(0,0,0)=-p[2 v+(1-2 v) / 2] \\
& \sigma_{y}(0,0,0)=-p[2 v+(1-2 v) / 2] \\
& \sigma_{z}(0,0,0)=-p
\end{aligned}
$$

The point shear traction $\tau_{x z} \Delta x \Delta y$ at $(0,0,0)$ yields the Cerruti solution with 


$$
\begin{aligned}
& \sigma_{x}(x, y, z)=\frac{\tau_{x z} \Delta x \Delta y}{2 \pi}\left[\frac{3 x^{3}}{r_{\mathrm{s}_{2}}{ }^{3}}-(1-2 v)\left\{\frac{x}{r_{\mathrm{s}_{2}}{ }^{3}}-\frac{3 x}{r_{\mathrm{s}_{2}}\left(r_{\mathrm{s}_{2}}+z\right)^{2}}+\frac{x^{3}}{r_{\mathrm{s}_{2}}{ }^{3}\left(r_{\mathrm{s}_{2}}+z\right)^{2}}+\frac{2 x^{3}}{r_{\mathrm{s}_{2}}{ }^{2}\left(r_{\mathrm{s}_{2}}+z\right)^{3}}\right\}\right] \\
& \sigma_{y}(x, y, z)=\frac{\tau_{x z} \Delta x \Delta y}{2 \pi}\left[\frac{3 x y^{2}}{r_{\mathrm{s}_{2}}{ }^{5}}-(1-2 v)\left\{\frac{x}{r_{\mathrm{s}_{2}}{ }^{3}}-\frac{x}{r_{\mathrm{s}_{2}}\left(r_{\mathrm{s}_{2}}+z\right)^{2}}+\frac{x y^{2}}{r_{\mathrm{s}_{2}}{ }^{3}\left(r_{\mathrm{s}_{2}}+z\right)^{2}}+\frac{2 x y^{2}}{r_{\mathrm{s}_{2}}{ }^{2}\left(r_{\mathrm{s}_{2}}+z\right)^{3}}\right\}\right] \\
& \sigma_{z}(x, y, z)=\frac{\tau_{x z} \Delta x \Delta y}{2 \pi} \frac{3 x z^{2}}{r_{\mathrm{s}_{2}}{ }^{5}} \\
& \tau_{x y}(x, y, z)=\frac{\tau_{x z} \Delta x \Delta y}{2 \pi}\left[\frac{3 x^{2} y}{r_{\mathrm{s}_{2}}{ }^{2}}-(1-2 v)\left\{-\frac{y}{r_{\mathrm{s}_{2}}\left(r_{\mathrm{s}_{2}}+z\right)^{2}}+\frac{x^{2} y}{r_{\mathrm{s}_{2}}{ }^{3}\left(r_{\mathrm{s}_{2}}+z\right)^{2}}+\frac{2 x^{2} y}{r_{\mathrm{s}_{2}}{ }^{2}\left(r_{\mathrm{s}_{2}}+z\right)^{3}}\right\}\right] \\
& \tau_{y z}(x, y, z)=\frac{\tau_{x z} \Delta x \Delta y}{2 \pi} \frac{3 x^{2} z}{r_{\mathrm{s}_{2}}{ }^{5}}
\end{aligned}
$$

At the surface $\tau_{x z}$ and $\tau_{y z}$ are equal to the EHL shear tractions, Eq. (12). In Eqs A3 and A5

$$
\begin{aligned}
& r_{\mathrm{s}_{1}}{ }^{2}=x^{2}+y^{2} \\
& r_{\mathrm{s}_{2}}{ }^{2}=x^{2}+y^{2}+z^{2}
\end{aligned}
$$




\section{Appendix B. Material fatigue data}

The gear material in Fig. 1. followed Swedish standard SS 142506 with both fatigue and monotonic data in the literature. The specimen had been case carburized in accord with the gear example. Endurance data for rotating bending with two levels of compressive residual stresses were taken from Preston [72] and pulsating torsion results with and without residual stresses are reported by Alfredsson and Olsson [50]; see Fig. B1 where the residual stresses were included in the mean stresses. Monotonic experiments containing the ultimate tensile strength were performed by Alfredsson and Olsson [69]. A non-linear curve fit gave the Findley parameters: $\kappa=0.627$ and $\sigma_{\mathrm{eF}}=625 \mathrm{MPa}$ for the surface material. Fig. B1 shows the Findley prediction for uni-axial bending and torsion including the endurance limit $\sigma_{\mathrm{eb}}=692$ MPa without any mean stress.

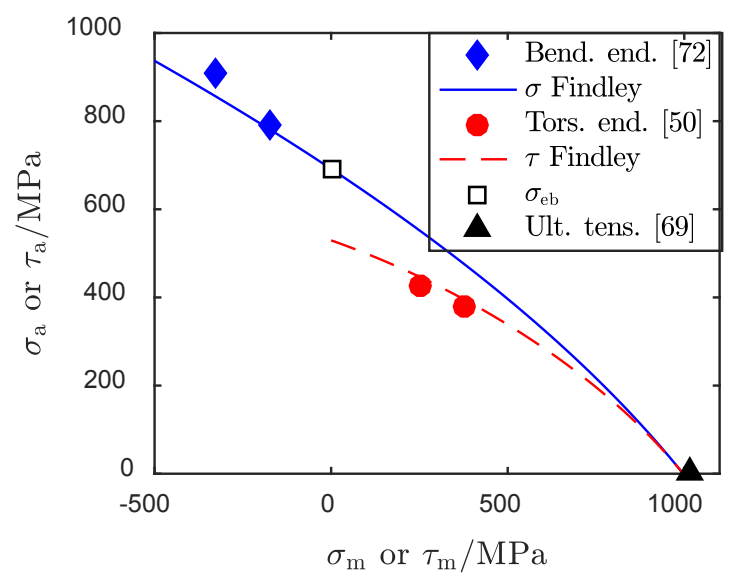

Fig. B1. Uni-axial fatigue data including the fitted Findley criterion. 


\section{Appendix C. Validation and Convergence study}

The model was verified against the results by Holmes et al. [68] for a ball on disk contact with a transverse ridge passing with the lubrication entrainment speed. Holmes et al. [68] validated their model against Venner [73]. The reference models were temperature independent. Hence, Eqs (7) and (9) became

$$
\rho=\rho_{0}\left[1+A_{1} p /\left(1+A_{2} p\right)\right]
$$

and

$$
\eta=\eta_{0} \exp \left(\frac{\alpha_{\text {Barus }} p_{0}}{z_{\mathrm{R}}}\left(-1+\left(1+\frac{p}{p_{0}}\right)^{z_{\mathrm{R}}}\right)\right)
$$

The line ridge was described by

$$
A_{\text {sh }}(X, T)=\Delta \cdot 10^{-10\left(\frac{X-X_{\mathrm{d}}}{\Omega_{\mathrm{x}}}\right)^{2}} \cos \left[2 \pi\left(X-X_{\mathrm{d}}\right) / \Omega_{\mathrm{x}}\right]
$$

The mechanical and numerical parameter values used for the validation are presented in Table C1. Fig. C1a compares results for the pressure, $P$, and the lubrication height, $H$, along the symmetry line at $Y=0$ when the ridge was located at $X_{\mathrm{d}}=0$. Based on Fig. C1a it was concluded that the present model converged to the correct solution.

Table C1. Mechanical and numerical parameters describing the reference simulations [68].

\begin{tabular}{lccc}
\hline \multicolumn{1}{c}{ Parameter } & Symbol & Value & Unit \\
\hline Entrainment speed & $u_{\mathrm{m}}$ & 0.01075 & $\mathrm{~m} / \mathrm{s}$ \\
Hertz pressure & $p_{\mathrm{Hertz}}$ & 0.54 & $\mathrm{GPa}$ \\
Half Hertz contact width & $b$ & $1.84 \cdot 10^{-1}$ & $\mathrm{~mm}$ \\
Equivalent elastic modulus & $E^{\prime}$ & 117 & $\mathrm{GPa}$ \\
Equivalent radius & $r^{\prime}$ & 12.7 & $\mathrm{~mm}$ \\
Pressure-viscosity coefficient & $a_{\mathrm{Barus}}$ & 22 & $\mathrm{GPa}^{-1}$ \\
Reference viscosity & $\eta_{0}$ & 1.22 & $\mathrm{~Pa} \cdot \mathrm{s}$ \\
Reference pressure & $p_{0}$ & 198 & $\mathrm{MPa}$ \\
Pressure-viscosity exponent & $Z_{\mathrm{R}}$ & 0.68 & - \\
Density coeff. & $A_{1}$ & 0.576 & $\mathrm{GPa}^{-1}$ \\
Density coeff. & $A_{2}$ & 1.69 & $\mathrm{GPa}^{-1}$ \\
Ridge height & $\delta$ & 0.2 & $\mu \mathrm{m}$ \\
Ridge wavelength & $\omega_{\mathrm{X}}$ & 129 & - \\
Inlet position & $X_{0}$ & -2.5 & - \\
Outlet position & $X_{\mathrm{e}}$ & 1.5 & - \\
Number of nodes & $N_{X} \cdot N_{Y}$ & $257 \cdot 257$ & - \\
Time step & $\Delta T$ & $7.8 \cdot 10^{-3}$ & - \\
Dimensionless speed parameter & $U$ & $8.8 \cdot 10^{-12}$ & - \\
Dimensionless material & $G$ & $2.6 \cdot 10^{3}$ & \\
parameter & & & \\
\hline
\end{tabular}


The discretization analysis for the present model was performed with the data presented in Table 1 and Table 2. The space discretization comprised a rolling smooth cylinder against a flat surface. The minimum and central film thicknesses in Fig. C1b and maximum EHL pressure in Fig. C1c are plotted against the number of nodes in the rolling direction, $N_{x}$. Holmes et al. [68] show in a similar graph for a point contact that the solutions for the film thickness converge with increasing node numbers. Based on Fig. C1b and Holmes et al., $N_{x} \cdot N_{y}=201 \cdot 97$ nodes was judged as sufficiently accurate for the present cylinder contact and that the increased solution time for doubled node numbers in both the $x$ - and $y$ directions could not be motivated.

The time discretization was investigated for the current transient problem of a rolling cylinder with an axial-symmetric asperity. $N_{x} \cdot N_{y}=201 \cdot 97$ nodes were used. Fig. C1c shows the pressure profiles and the film thicknesses when the asperity is at $X_{\mathrm{d}}=0.0$. Based on Fig. C1c, $N_{t}=402$ was judged to give a sufficiently accurate resolution. Holmes et al. performed a similar investigation for a point contact with a transverse ridge. They found that the CrankNicholson scheme and $N_{x} / N_{t}=0.5$ gave good agreement with the reference solution, which gave further support for using $N_{x} / N_{t}=0.5$ here.
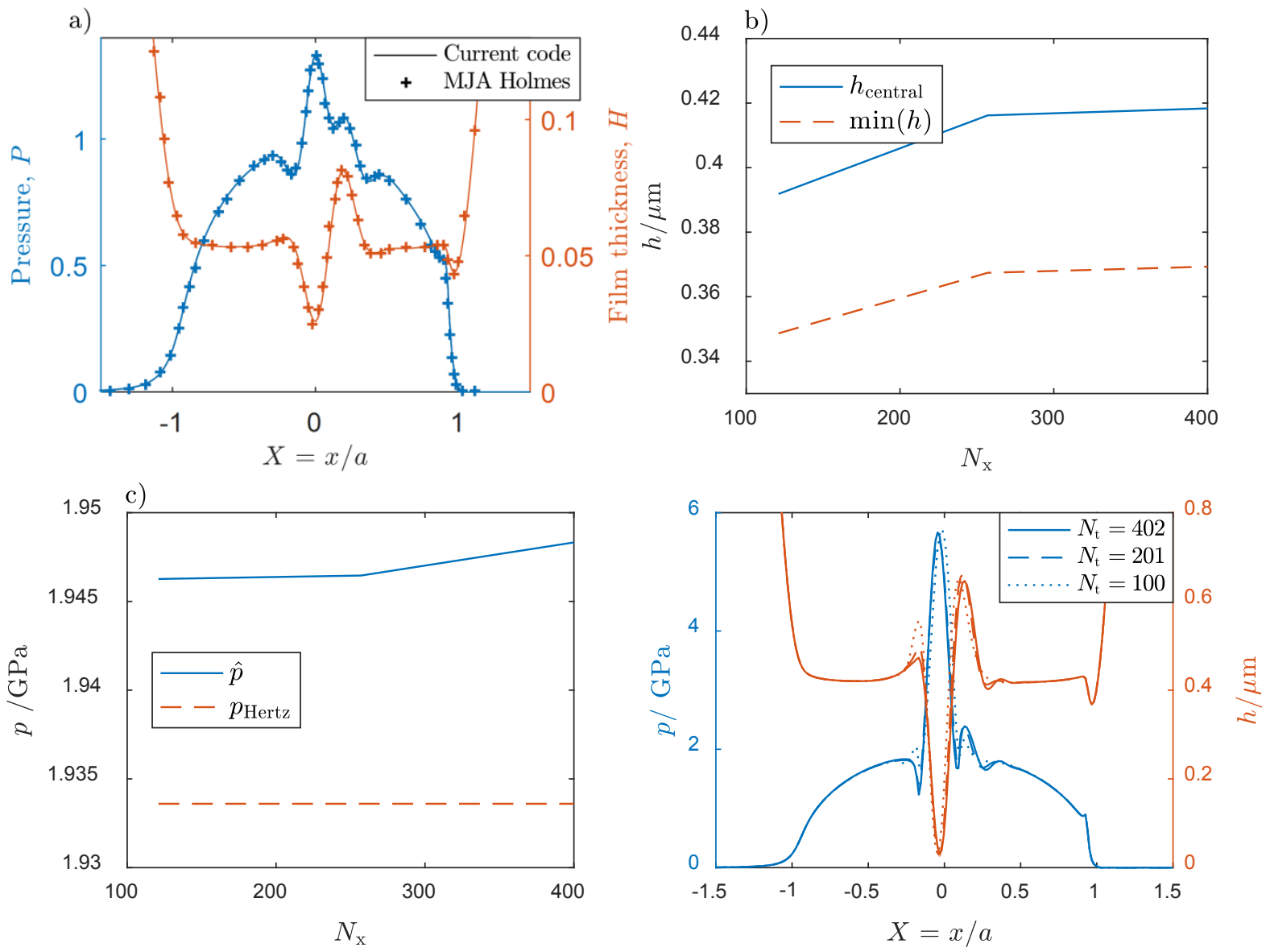

Fig. C1. a) Model validation against Holmes et al. [68] for transverse ridge passing a rolling sphere with parameters in Table C1. Convergence study for spatial resolution of $h$ in b) and $p$ in c) for rolling smooth cylinder. d) Time discretization study of over-rolling with asperity at $X_{\mathrm{d}}=0.0$. Data from Table 1 and Table 2. 